

\title{
Fast evaluation of radial basis functions: Methods for four-dimensional polyharmonic splines
}

\author{
R. K. Beatson* \\ J. B. Cherrie* \\ D. L. Ragozin ${ }^{\dagger}$
}

27 April, 2000

\begin{abstract}
As is now well known for some basic functions $\phi$, hierarchical and fast multipole like methods can greatly reduce the storage and operation counts for fitting and evaluating radial basis functions. In particular for spline functions of the form.

$$
s(x)=p(x)+\sum_{k=1}^{N} d_{k} \phi\left(\left|x-x_{k}\right|\right),
$$

$p$ a low degree polynomial and certain choices of $\phi$, the cost of a single extra evaluation can be reduced from $\mathcal{O}(N)$ to $\mathcal{O}(\log N)$, or even $\mathcal{O}(1)$, operations and the cost of a matrix-vector product (i.e., evaluation at all centres) can be decreased from $\mathcal{O}\left(N^{2}\right)$ to $\mathcal{O}(N \log N)$, or even $\mathcal{O}(N)$, operations.

This paper develops the mathematics required by methods of these types for polyharmonic splines in $\mathcal{R}^{4}$. That is for splines $s$ built from a basic function from the list $\phi(r)=r^{-2}$ or $\phi(r)=r^{2 n} \ln (r), n=0,1, \ldots$. We give appropriate far and near field expansions, together with corresponding error estimates, uniqueness theorems, and translation formulae.

A significant new feature of the current work is the use of arguments based on the action of the group of non-zero quaternions, realised as $2 \times 2$ complex matrices

$$
\mathcal{H}_{0}^{1}=\left\{x=\left[\begin{array}{cc}
z & w \\
-\bar{w} & \bar{z}
\end{array}\right]:|z|^{2}+|w|^{2}>0\right\}
$$

acting on $\mathcal{C}^{2}=\mathcal{R}^{4}$. Use of this perspective allows us to give a relatively efficient development of the relevant spherical harmonics and their properties.
\end{abstract}

\section{Introduction.}

In a large scale comparison of methods for interpolating 2D scattered data Franke [10] identified radial basis functions as one of the most promising methods. These are functions of the form

$$
s(\cdot)=p(\cdot)+\sum_{k=1}^{N} d_{k} \phi\left(\left|\cdot-x_{k}\right|\right)
$$

where $p$ is a low degree polynomial, and the basic function $\phi$ is usually of non-compact support [17]. Statisticians have also successfully employed radial basis functions fitted by generalised cross validation to smoothing noisy data, e.g., in modelling rainfall distribution across Australia [13]. However, widespread adoption of these techniques has been delayed by their apparent extreme computational cost. For example, conventional methods for fitting by interpolation require $\mathcal{O}\left(N^{2}\right)$

*Department of Mathematics and Statistics, University of Canterbury, Private Bag 4800, Christchurch, New Zealand. This research was partially supported by PGSF subcontract DRF601.

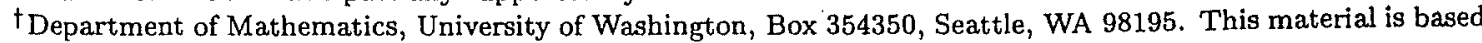
upon work supported by the National Science Foundation Grant No. DMS-9972004. 
storage and $\mathcal{O}\left(N^{3}\right)$ arithmetic operations, where $N$ is the number of data points. This makes computations when $N$ exceeds 10,000 totally impractical.

These storage and operation counts had led many researchers to incorrectly conclude that the computations are all but impossible. Sibson and Stone $[20,1991]$ talking about problems with 50,000 to 100,000 data sites state: "We believe such problems will indefinitely remain beyond the scope of thin-plate splines." Also Flusser [9, 1992], in the context of warping digital images, comments on the computational complexity of evaluation of thin-plate splines, concluding that "their direct use has extreme computing complexity and is not suitable for practical applications."

Recent algorithmic advances involving hierarchical and fast multipole like methods have invalidated the comments noted above, at least for 2 and 3 dimensional data, see $[3,4,7,8,11]$. These algorithmic developments employ far field and near field expansions to reduce the computational cost of evaluating an $N$ centre polyharmonic radial basis function at $m \geq N$ points to $\mathcal{O}(m \log N)$ or even $\mathcal{O}(m)$ operations, at least in $\mathcal{R}^{2}$ and $\mathcal{R}^{3}$. Furthermore, interpolatory fitters have been developed which can solve the interpolation problem for such splines in $\mathcal{O}(N)$ storage and $\mathcal{O}(N \log N)$ arithmetic operations. These iterative fitters use the hierarchical evaluators as fast matrix-vector multipliers and can fit interpolatory thin-plate splines to 100,000 data points in a few minutes on relatively inexpensive workstations.

Polyharmonic splines in $\mathcal{R}^{4}$ are spline functions of the form (1.1) where the basic function $\phi$ is a member of the list

$$
\phi(r)=\left\{\begin{array}{l}
r^{-2}, \\
r^{2 n} \ln (r), \quad n=0,1, \ldots
\end{array}\right.
$$

Interpolatory splines of this type minimize suitable energy semi-norms, as do their analogues in lower dimensions. For example, the functional

$$
I(s)=\int_{\mathcal{R}^{4}} \sum_{|\alpha|=3}\left(\begin{array}{l}
3 \\
\alpha
\end{array}\right)\left(\left(D^{\alpha} s\right)(x)\right)^{2} d x
$$

is minimized over all suitably smooth functions, satisfying the interpolation conditions

$$
s\left(x_{k}\right)=f\left(x_{k}\right), \quad k=1, \ldots, N,
$$

if and only if $s$ is the triharmonic spline

$$
s(\cdot)=p_{2}(\cdot)+\sum_{k=1}^{N} d_{k}\left|\cdot-x_{k}\right|^{2} \ln \left|\cdot-x_{k}\right|,
$$

where $p_{2} \in \pi_{2}^{4}$ (the space of quadratics in 4 variables), and the coefficients $\left\{d_{k}\right\}$, and those of the polynomial $p_{2}$, are determined by the interpolation conditions (1.3) along with the orthogonality conditions

$$
\sum_{k=1}^{N} d_{k} q\left(x_{k}\right)=0, \quad \text { for all } q \in \pi_{2}^{4} .
$$

Thus polyharmonic splines in $\mathcal{R}^{4}$ can be expected to be highly successful approximators and interpolators, as experience has shown the polyharmonic splines in lower dimensions to be. However, meaningful data sets in $\mathcal{R}^{4}$ can be expected to have many points. Hence, the development of fast evaluation and fitting methods is almost a prerequisite to the use of polyharmonic splines in $\mathcal{R}^{4}$. Motivated by this we will develop the analytic underpinnings of a fast hierarchical and a fast multipole like method for polyharmonic splines in $\mathcal{R}^{4}$. There are many potential applications of these fast methods. One possible application to data mining is estimating the probability of some attribute, such as early death due to heart attack or the filing of a fraudulent tax return, by a regression spline depending on four predictor variables. An application to environmental engineering is modelling the concentration of some chemical, or pollutant, as a function of position and time. Turk \& O'Brien [21] suggest using polyharmonic splines in $\mathcal{R}^{4}$ for shape transformation, or morphing, of implicitly defined $3 D$-surfaces. 
The core ideas underlying hierarchical, and fast multipole methods, are beautifully simple. They will be described in this paragraph. First, one needs to accept that only a certain finite precision is necessary. This allows the use of approximations. Second, a suitable far field expansion about 0 must be known for the shifted basic function $\phi\left(\left|\cdot-x_{<}\right|\right)$. Here, a far field expansion is a series expansion in which the influence of the source point $x_{<}$, and the evaluation point $x$, separates. Furthermore, the series should converge at a geometric rate at all points $x$ with $|x|$ sufficiently large compared to $\left|x_{<}\right|$. Associate with any region $T$ in $\mathcal{R}^{d}$ the part of the RBF due to sources in $T$,

$$
s_{T}=\sum_{k: x_{k} \in T} d_{k} \phi\left(\left|\cdot-x_{k}\right|\right) .
$$

Approximate $s_{T}$ by a truncated far field expansion $r_{T}$, chosen to have appropriate accuracy at all evaluation points $x$ sufficiently far from the centre of $T$. If the number of centres $x_{k}$ in $T$ is large compared to the number of terms in the far field series, it will be quicker to approximately evaluate $s_{T}(x)$ by evaluating the series $r_{T}(x)$, than by evaluating $s_{T}(x)$ directly.

The idea, described above, of using an approximating series when it is faster to do so, lies at the heart of hierarchical methods. Its application is organised by using also a hierarchical subdivision of space. This subdivision determines for a given evaluation point $x$ which parts of $s$, that is which $s_{T}$ 's, should be approximated by the corresponding far field series $r_{T}$, and which parts are to be evaluated directly. In order to be more explicit about the algorithmic organisation of the evaluation process, suppose for the moment that space is subdivided into a binary tree of rectangular panels. The root panel will be chosen to contain all the centres, and the children of each parent will be formed by splitting the parent with a hyperplane. Associate with every panel $T$, the RBF $s_{T}$, far field approximation $r_{T}$, and a distance from the centre of $T$ at which $r_{T}$ gives a sufficiently accurate approximation to $s_{T}$. Then approximate evaluation of $s(x)$ can be performed by a recursive descent of the tree. The actions to be taken when panel $T$ is encountered during this descent being as follows:

- If $x$ is sufficiently far from $T$, that is if the distance from $x$ to the centre of $T$ is large enough, then the approximation to $s(x)$ is incremented with the approximation $r_{T}(x)$ to $s_{T}(x)$.

- If $T$ is childless the approximation to $s(x)$ is incremented by the directly calculated value of $s_{T}(x)$.

- Otherwise the process descends to the children of $T$.

We turn now from algorithmic matters to the analytic underpinnings of a generic fast multipole method. Results of the following nature are required for the basic function $\phi$ being used:

- The existence of a rapidly converging far field expansion, centred at 0 , for the shifted basic function $\phi\left(\left|\cdot-x_{<}\right|\right)$, e.g., Lemmas 4.1 and 4.4 .

- Error bounds that determine how many terms are required in each expansion to achieve a specified accuracy, e.g., Theorems 4.2 and 4.10 .

- Efficient recurrence relations for computing the coefficients of the expansions, e.g., Lemmas 3.3 and 3.4.

- Uniqueness results that justify indirect translation of expansions, thus allowing the expansions of parent panels to be calculated quickly from those of children, e.g., Lemmas 5.1 and 5.2 .

- Formulae for efficiently converting a far field expansion to a rapidly convergent local expansion, e.g., Theorem 6.1.

This paper provides appropriate mathematics for polyharmonic radial basis functions on $\mathcal{R}^{4}$. That is for functions of the form (1.1) where $\phi$ is given by (1.2). 
Our discussion above outlines the analytic and algorithmic underpinnings of hierarchical and fast multipole methods. More detailed discussions may be found in the original paper of Greengard and Rokhlin [11], or the introductory short course [3]. Previous papers concerning fast multipole and related methods for fast evaluation of radial basis functions include $[4,5,6]$.

A significant technique in our development in this paper is the use of a group action perspective. In particular of arguments based on the action of the group of non-zero quaternions, realized as $2 \times 2$ complex matrices

$$
\mathcal{H}_{0}^{1}=\left\{x=\left[\begin{array}{cc}
z & w \\
-\bar{w} & \bar{z}
\end{array}\right]:|z|^{2}+|w|^{2}>0\right\}
$$

acting on $\mathcal{C}^{2}=\mathcal{R}^{4}$. We develop almost all the (simple) details needed for these arguments without relying on other presentations of the possibly unfamiliar group representation theory. Use of this perspective allows us to give a relatively efficient development of the relevant spherical harmonics and their properties. See $[18,19]$ for related analyses of spherical harmonics and their approximation properties. Our work has also been influenced by the elegant and concise treatment of Epton and Dembart [8] of the analogous expansions for the three-dimensional fast multipole method.

This paper is organised as follows. Section 2 concerns some of the properties of polyharmonic functions on $\mathcal{R}^{4}$-including realisations of $\mathcal{R}^{4}$ and representations of $\mathcal{H}_{0}^{1}$. It also introduces the inner and outer functions (spherical harmonics) that form the basis of our far field expansions. Section 3 developes a number of properties of these functions that can be applied to far field "expansions. These include recurrence formulae, derivative formulae and symmetries. Section 4 contains the main results on the far field expansions themselves and the associated error bounds. Section 5 developes the uniqueness results that allow the far field expansions to be computed indirectly and economically via the translation theory of Section 6 . Section 6 also contains the outer-to-inner and inner-to-inner translation formulae needed to approximate far field series by local Taylor series.

\section{Polyharmonic functions on $\mathcal{R}^{4}$.}

We will represent a non-zero $x \in \mathcal{R}^{4}$ in three different ways:

$$
x=\left(\begin{array}{l}
x_{1} \\
x_{2} \\
x_{3} \\
x_{4}
\end{array}\right) \quad \text { or }[z, w] \quad \text { or }\left[\begin{array}{cc}
z & w \\
-\bar{w} & \bar{z}
\end{array}\right]
$$

where $z=x_{1}+\mathbf{i} x_{2}, w=x_{3}+\mathbf{i} x_{4}$ and $x_{1}, \ldots, x_{4} \in \mathcal{R}$. The first of these is just an element of $\mathcal{R}^{4}$, the second is as an element of $\mathcal{C}^{2}$ and the last as an element of the punctured quaternion (Hamiltonian) space

$$
\mathcal{H}_{0}^{1}=\left\{x=\left[\begin{array}{cc}
z & w \\
-\bar{w} & \bar{z}
\end{array}\right]:|z|^{2}+|w|^{2}>0\right\} .
$$

Note that for elements of $\mathcal{H}_{0}^{1}$ the classical adjoint or adjugate and the Hermitian adjoint coincide and

$$
x^{-1}=x^{*} / \operatorname{det}(x) \text {. }
$$

We are primarily interested in $\mathcal{R}^{4}$ with the usual inner product,

$$
\left\langle x, x_{<}\right\rangle=x_{1} x_{<1}+x_{2} x_{<2}+x_{3} x_{<3}+x_{4} x_{<4}=|x|\left|x_{<}\right| \cos \theta,
$$

where $|\cdot|$ is the 2 -norm for $\mathcal{R}^{4}$. In terms of the $\mathcal{C}^{2}$ realisation of $\mathcal{R}^{4}$ this becomes

$$
\left\langle x, x_{<}\right\rangle=\Re\left(z \overline{z_{<}}+w \overline{w_{<}}\right)=\frac{1}{2}\left(z \overline{z_{<}}+w \overline{w_{<}}+z_{<} \bar{z}+w_{<} \bar{w}\right)
$$

and, in terms of the matrix realisation $\mathcal{H}_{0}^{1}$,

$$
\left\langle x, x_{<}\right\rangle=\frac{1}{2} \operatorname{Tr}\left(x^{*} x_{<}\right)=\frac{1}{2}|x|^{2} \operatorname{Tr}\left(x^{-1} x_{<}\right)=\frac{1}{2} \operatorname{Tr}\left(x_{<}{ }^{*} x\right),
$$


where $\operatorname{Tr}$ is the trace. Note that this inner product gives the norm

$$
|x|^{2}=x_{1}^{2}+x_{2}^{2}+x_{3}^{2}+x_{4}^{2}=z \bar{z}+w \bar{w}=\operatorname{det}(x) .
$$

We also require an inner product for functions on the unit ball $B=\left\{x \in \mathcal{R}^{4}:|x| \leq 1\right\}$. For $f, g \in L^{2}(B)$ we define their inner product by

$$
(f, g)=\int_{|\xi| \leq 1} f(\xi) \overline{g(\xi)} d \xi .
$$

We will also use this pairing for other functions $f$ and $g$ on $B$ with $f \bar{g} \in L^{1}(B)$. Furthermore, we will also require the subspaces of $C\left(\mathcal{R}^{4}\right)$ defined by

$$
\mathcal{H}_{m}=\left\{p\left(\left[z_{1}, z_{2}\right]\right): p \text { is a homogeneous polynomial of degree } m \text { in } z_{1}, z_{2}\right\}, \quad m \in \mathcal{N}_{0},
$$

where $\mathcal{N}_{0}$ is the non-negative integers.

\subsection{Irreducible representations of $\mathcal{H}_{0}^{1}$-spherical harmonics.}

In this section we will develop irreducible representations of $\mathcal{H}_{0}^{1}$ in $\mathcal{H}_{m}$. Our purpose for doing so is that the coefficient functions of these representations will eventually be seen to form a very computationally convenient basis for the harmonic polynomials in $\mathcal{R}^{4}$. In fact when these coefficient functions are multiplied by $|x|^{2 \ell}, \ell=0, \ldots, k$ they yield a basis for all $(k+1)$-harmonic polynomials in $\mathcal{R}^{4}$. We note in particular the simple form of the addition formulae (Lemma 2.9), the recurrence relation (Lemma 3.3), and the dual basis (Lemma 3.10), to come.

Most of the relevant representation theory and mathematical physics literature is focused on rotations of $\mathcal{S}^{2}$ or $\mathcal{S}^{3}$ and therefore considers representations of

$$
S U(2)=\left\{x \in \mathcal{H}_{0}^{1}: \operatorname{det}(x)=|x|^{2}=1\right\}=\mathcal{S}^{3} .
$$

However, in the context of far field expansions it is convenient to work instead with all of $\mathcal{H}_{0}^{1}$ to take into account both the scaling by $|x|$ and rotation by elements of $S U(2)$. This leads to some differences, most importantly the character functions now depend on the norm of $x$ as well as the angle $\theta$ between $x$ and the north pole $[1,0]$. Other differences include the formula for the product of two character functions and the addition formulae.

Definition 2.1. Given a group $G$, a representation $T: G \rightarrow \mathrm{GL}(V)$ of $G$ on $V$ is an operator valued map that satisfies $T(g \cdot h)=T(g) T(h)$. A representation $T$ of $G$ on $V$ is irreducible if the only subspaces of $V$ that are invariant under $T(g)$ for all $g \in G$ are $\{0\}$ and $V$.

We define representations $T_{m}(x)$ of $\mathcal{H}_{0}^{1}$ in the spaces $\mathcal{H}_{m}$ given by

$$
\begin{aligned}
T_{m}(x) p\left(\left[z_{1}, z_{2}\right]\right) & =p\left(\left[z_{1}, z_{2}\right] x\right) \\
& =p\left(\left[z_{1} z-z_{2} \bar{w}, z_{1} w+z_{2} \bar{z}\right]\right) .
\end{aligned}
$$

Note that since $\mathcal{H}_{m}$ is embedded in the space of functions on $\mathcal{H}^{1}$ (or even on $\mathcal{H}_{0}^{1}$ ), this representation is just the restriction of the right action defined by

$$
(x \cdot f)\left(\left[z_{1}, z_{2}\right]\right)=f\left(\left[z_{1}, z_{2}\right] x\right), \quad x \in \mathcal{H}_{0}^{1} .
$$

If we put a (Hilbert space) norm on these functions via (2.2), i.e.,

$$
\|f\|^{2}=(f, f)=\int_{|\xi| \leq 1}|f(\xi)|^{2} d \xi
$$

then the rotation invariance of Lebesgue measure implies that $\|x \cdot f\|=\|f\|$, whenever $|x|=1$, i.e., whenever $x \in \mathcal{S}^{3}$. Thus

$$
\left(T_{m}(x) f, T_{m}(x) f\right)=(f, f)
$$

for all functions $f \in L^{2}(B)$, and $T_{m}(x)$, as an operator, is unitary if $|x|=1$. The reader is cautioned that the matrix realisation of $T_{m}(x)$ to come is not unitary, but can be scaled to be so (see Lemma 2.5). 
Lemma 2.2. The representations (2.3) are irreducible.

Proof. Assume there is a subspace $V \subset \mathcal{H}_{m}$ that is invariant under $T_{m}(x)$ for all $x \in \mathcal{H}_{0}^{1}$. Then $V$ is invariant under $T_{m}(x)$ if we restrict attention to $x \in S U(2) \subset \mathcal{H}_{0}^{1}$. Since the representations $T_{m}$ of $S U(2)$ are irreducible [16, pp. 208-211], it follows that either $V=\{0\}$ or $V=\mathcal{H}_{m}$ and hence that the representations $T_{m}$ are irreducible.

The monomials

$$
e_{k}^{m}\left(\left[z_{1}, z_{2}\right]\right)=z_{1}^{m-k} z_{2}^{k}, \quad 0 \leq k \leq m
$$

form a basis for $\mathcal{H}_{m}$. The operators $T_{m}(x)$ have a matrix realisation once this basis for $\mathcal{H}_{m}$ is chosen. The elements of these matrices will be denoted $t_{i, j}^{m}$ and are given by

$$
\begin{aligned}
t_{i, j}^{m}(x) & =t_{i, j}^{m}(z, w) \\
& =\text { coefficient of } e_{i}^{m} \text { in } T_{m}(x) e_{j}^{m} \\
& =\text { coefficient of } z_{1}^{m-i} z_{2}^{i} \text { in }\left(z_{1} z-z_{2} \bar{w}\right)^{m-j}\left(z_{1} w+z_{2} \bar{z}\right)^{j} .
\end{aligned}
$$

Equivalently,

$$
\sum_{i=0}^{m} t_{i, j}^{m}(z, w) z_{1}^{m-i} z_{2}^{i}=\left(z_{1} z-z_{2} \bar{w}\right)^{m-j}\left(z_{1} w+z_{2} \bar{z}\right)^{j} .
$$

For $m=0, t_{0,0}^{0}(x)=1$, while for $m=1$, from $(2.4)$

$$
\begin{aligned}
t_{0,0}^{1}(z, w) & =z, & t_{0,1}^{1}(z, w) & =w, \\
t_{1,0}^{1}(z, w) & =-\bar{w}, & t_{1,1}^{1}(z, w) & =\bar{z},
\end{aligned}
$$

or in matrix terms

$$
\left[T_{1}(x)\right]=x .
$$

An immediate consequence of this choice of basis is the following lemma.

Lemma 2.3. Treated as matrices, $T_{m}(z, 0)$ is a diagonal matrix and $T_{m}(0, w)$ is an anti-diagonal matrix. Specifically these matrices have entries

$$
t_{i, j}^{m}(z, 0)= \begin{cases}0, & i \neq j \\ z^{m-i} \bar{z}^{i}, & i=j\end{cases}
$$

and

$$
t_{i, j}^{m}(0, w)= \begin{cases}0, & i \neq m-j \\ w^{m-i}(-\bar{w})^{i}, & i=m-j\end{cases}
$$

The basis elements $e_{k}^{m}$ for $\mathcal{H}_{m}$ are orthogonal with respect to the inner product (2.2). In fact the exact norms for the basis elements $e_{k}^{m}$ and thus the row and column scalings to get unitary matrices are easily computed.

Lemma 2.4. The basis functions $e_{k}^{m}$ are orthogonal with inner products given by

$$
\left(e_{k}^{m}, e_{k^{\prime}}^{m^{\prime}}\right)=\int_{|\xi| \leq 1} e_{k}^{m}(\xi) \overline{e_{k^{\prime}}^{m^{\prime}}(\xi)} d \xi=\delta_{m, m^{\prime}} \delta_{k, k^{\prime}} \pi^{2} \frac{k !(m-k) !}{(m+2) !}
$$

Proof. Introduce polar coordinates $\left(r_{1}, \theta_{1}\right)$ and $\left(r_{2}, \theta_{2}\right)$ in the $z_{1}$ and $z_{2}$ planes (where $\xi=\left[z_{1}, z_{2}\right]$ ).

$$
\begin{aligned}
\left(e_{k}^{m}, e_{k^{\prime}}^{m^{\prime}}\right) & =\int_{|\xi| \leq 1} e_{k}^{m}(\xi) \overline{e_{k^{\prime}}^{m^{\prime}}(\xi)} d \xi \\
& =\int_{0}^{1} \int_{0}^{\sqrt{1-r_{1}^{2}}} \int_{0}^{2 \pi} \int_{0}^{2 \pi} r_{1}^{m-k} e^{\mathrm{i}(m-k) \theta_{1}} r_{2}^{k} e^{\mathrm{i} k \theta_{2}} \\
& \quad \times r_{1}^{m^{\prime}-k^{\prime}} e^{-\mathrm{i}\left(m^{\prime}-k^{\prime}\right) \theta_{1}} r_{2}^{k^{\prime}} e^{-\mathrm{i} k^{\prime} \theta_{2}} d \theta_{2} d \theta_{1} r_{2} d r_{2} r_{1} d r_{1}
\end{aligned}
$$




$$
\begin{aligned}
& =(2 \pi)^{2} \delta_{k, k^{\prime}} \delta_{m, m^{\prime}} \int_{0}^{1} \int_{0}^{\sqrt{1-r_{1}^{2}}} r_{1}^{2(m-k)+1} r_{2}^{2 k+1} d r_{2} d r_{1} \\
& =(2 \pi)^{2} \delta_{k, k^{\prime}} \delta_{m, m^{\prime}} \int_{0}^{1} r_{1}^{2(m-k)+1} \frac{\left(1-r_{1}^{2}\right)^{k+1}}{2 k+2} d r_{1} \\
& =\pi^{2} \delta_{k, k^{\prime}} \delta_{m, m^{\prime}} \mathrm{B}(m-k+1, k+2) /(k+1) \\
& =\pi^{2} \delta_{k, k^{\prime}} \delta_{m, m^{\prime}} \frac{(m-k) !(k+1) !}{(m+2) !(k+1)}
\end{aligned}
$$

where $\mathrm{B}$ is the Beta function $\mathrm{B}(n, m)=\Gamma(n) \Gamma(m) / \Gamma(n+m)$.

Since $T_{m}$ restricted to $\mathcal{S}^{3}$ acts in a norm preserving way on $\mathcal{H}_{m}$, we easily obtain the following matrix representation for $T_{m}\left(x^{-1}\right)$.

Lemma 2.5. There exist row and column scalings that make the matrices $T_{m}(x)$ unitary for $|x|=1$. Specifically

(i) The inverse of $T_{m}(x)$ is given by

$$
T_{m}\left(x^{-1}\right)=\left[t_{i, j}^{m}\left(x^{-1}\right)\right]=\left[|x|^{-2 m} \overline{t_{j, i}^{m}(x)}\left(\begin{array}{c}
m \\
i
\end{array}\right)\left(\begin{array}{c}
m \\
j
\end{array}\right)^{-1}\right],
$$

or equivalently via

$$
t_{i, j}^{m}\left(x^{*}\right)=t_{i, j}^{m}(\bar{z},-w)=\overline{t_{j, i}^{m}(z, w)}\left(\begin{array}{c}
m \\
i
\end{array}\right)\left(\begin{array}{c}
m \\
j
\end{array}\right)^{-1}
$$

(ii) The matrices

$$
U_{m}(x)=\left[t_{i, j}^{m}(x) \sqrt{\left(\begin{array}{c}
m \\
j
\end{array}\right)\left(\begin{array}{c}
m \\
i
\end{array}\right)^{-1}}\right]
$$

are unitary when $|x|=1$. For all $x \neq 0, U_{m}(x)^{-1}=|x|^{-2 m} U_{m}(x)^{*}$.

Proof. The definition of $t_{j, i}^{m}$ and the orthogonality of $\left\{e_{k}^{m}: k=0, \ldots, m\right\}$ implies

$$
\left(e_{j}^{m}, T_{m}(x) e_{i}^{m}\right)=\left(e_{j}^{m}, t_{j, i}^{m}(x) e_{j}^{m}\right)=\overline{t_{j, i}^{m}(x)}\left(e_{j}^{m}, e_{j}^{m}\right) .
$$

Since $T_{m}\left((x /|x|)^{-1}\right)$ preserves the inner product $(2.2)$,

$$
\begin{aligned}
\left(e_{j}^{m}, T_{m}(x) e_{i}^{m}\right) & =\left(T_{m}\left((x /|x|)^{-1}\right) e_{j}^{m}, T_{m}\left((x /|x|)^{-1}\right) T_{m}(x) e_{i}^{m}\right) \\
& =\left(|x|^{m} T_{m}\left(x^{-1}\right) e_{j}^{m},|x|^{m} e_{i}^{m}\right)=|x|^{2 m} t_{i, j}^{m}\left(x^{-1}\right)\left(e_{i}^{m}, e_{i}^{m}\right) .
\end{aligned}
$$

Equating (2.6) to (2.7) and solving, we obtain

$$
t_{i, j}^{m}\left(x^{-1}\right)=|x|^{-2 m} \overline{t_{j, i}^{m}(x)} \frac{\left(e_{j}^{m}, e_{j}^{m}\right)}{\left(e_{i}^{m}, e_{i}^{m}\right)} .
$$

Taking into account the previous lemma and the fact that $x^{-1}=[\bar{z},-w] /(z \bar{z}+w \bar{w})$, this gives the desired results.

Since it is easy to use the definitions to show the first row of each $T_{m}(x)$ is given by

$$
t_{0, j}^{m}(x)=t_{0, j}^{m}(z, w)=z^{m-j} w^{j}=e_{j}^{m}([z, w]),
$$

part of Lemma 2.4 shows that $\left\{t_{0, j}^{m}\right\}$ is orthogonal with respect to the inner product (2.2). In fact much more general (bi-)orthogonality facts are true for $t_{i, j}^{m}(x)$ and $\overline{t_{j, i}^{m}\left(x^{-1}\right)}$. These are related to the orthogonality properties of the irreducible unitary matrix representations of any compact group, such as $S^{3}$, as in [12, (27.19)]. But we prefer to present them in a slightly more general form which is closely related to the coordinate free proofs in [1, Chapter 3], particularly Proposition 3.15, Shur's Lemma, 3.22, and its corollary, 3.23. 


\section{Lemma 2.6.}

(i) (Shur's Lemma) For any $(m+1) \times(m+1)$ matrix $A$

$$
\widetilde{A}=\int_{0<|x| \leq 1} T_{m}\left(x^{-1}\right) A T_{m}(x) d x=c I, \quad c=\frac{\operatorname{vol}\{|x| \leq 1\}}{m+1} \operatorname{Tr}(A)=\frac{\pi^{2} / 2}{m+1} \operatorname{Tr}(A) .
$$

(ii) The set

$$
\left\{\frac{(m+1)}{\left(\pi^{2} / 2\right)}\left(\begin{array}{c}
m \\
j
\end{array}\right)\left(\begin{array}{c}
m \\
i
\end{array}\right)^{-1}|\cdot|^{-2 m} t_{i, j}^{m}(\cdot)=\frac{m+1}{\pi^{2} / 2} \overline{t_{j, i}^{m}(\cdot-1)}, i, j=0, \ldots, m\right\}
$$

is biorthogonally dual to $\left\{t_{i, j}^{m}(\cdot), i, j=0, \ldots, m\right\}$ with respect to the pairing (2.2). That is,

$$
\int_{0<|x| \leq 1} \frac{m+1}{\pi^{2} / 2} t_{i^{\prime}, j^{\prime}}^{m}(x) t_{j, i}^{m}\left(x^{-1}\right) d x=\delta_{i, i^{\prime}} \delta_{j, j^{\prime}}
$$

(iii) The first two parts are also true when $\{0<|x| \leq 1\}$ is replaced by $\mathcal{S}^{3}$ and "vol" is replaced by "surface area" (so $\pi^{2} / 2$ is replaced by $2 \pi^{2}$ ).

Proof. For (i), let $y \in \mathcal{S}^{3}$ be arbitrary. Then

$$
\widetilde{A} T_{m}(y)=\int_{0<|x| \leq 1} T_{m}\left(x^{-1}\right) A T_{m}(x y) d x=\int_{0<|x| \leq 1} T_{m}\left(y x^{-1}\right) A T_{m}(x) d x=T_{m}(y) \widetilde{A}
$$

since $x \mapsto x y^{-1}$ leaves Lebesgue measure invariant. Let $c$ be any eigenvalue for $\widetilde{A}$ with $v$ an associated eigenvector. From (2.8) $T_{m}(y) v$ is also an eigenvector for the same eigenvalue $c$. By the irreducibility of $T_{m}$, span $\left\{T_{m}(y) v: y \in \mathcal{S}^{3}\right\}=\mathcal{H}_{m}$. Thus $\widetilde{A} v=c v$ for all vectors $v$ and it follows that $\widetilde{A}=c I$.

To get the formula for $c$, take the trace of all terms. Then move the linear functional Tr inside the integral and use $\operatorname{Tr}\left(T_{m}\left(x^{-1}\right) A T_{m}(x)\right)=\operatorname{Tr}(A)$ to obtain

$$
\operatorname{Tr}(c I)=(m+1) c=\int_{0<|x| \leq 1} \operatorname{Tr}(A) d x=\operatorname{vol}\{0<|x| \leq 1\} \operatorname{Tr}(A) .
$$

For (ii) substitute $A=E_{i, i^{\prime}}=\left[\delta_{i, j} \delta_{j^{\prime}, i^{\prime}}\right]$ into (i), i.e., use the matrix $A$ with 1 at row $i$, column $i^{\prime}$. Then

$$
T_{m}\left(x^{-1}\right) E_{i, i^{\prime}} T_{m}(x)=\left[t_{j, i}^{m}\left(x^{-1}\right) t_{i^{\prime}, j^{\prime}}^{m}(x)\right]
$$

Since $\operatorname{Tr} E_{i, i^{\prime}}=\delta_{i, i^{\prime}}$, (i) yields

$$
\left[\int_{0<|x| \leq 1} t_{j, i}^{m}\left(x^{-1}\right) t_{i^{\prime}, j^{\prime}}^{m}(x) d x\right]=\frac{\pi^{2} / 2}{m+1} \delta_{i, i^{\prime}}\left[\delta_{j, j^{\prime}}\right] .
$$

For (iii) repeat the proofs with the ball replaced by the sphere. Or, more simply, just note that if $d \Omega$ denotes the standard "surface" measure on $S^{3}$, then integration in spherical coordinates is with respect to $|x|^{3} d \Omega(x /|x|) d|x|$. Then, due to the homogeneity of $T_{m}$, (i) becomes

$$
\begin{aligned}
\int_{\mathcal{S}^{3}} \int_{|x|=0}^{|x|=1} T_{m}\left((x /|x|)^{-1}\right) A T_{m}(x /|x|) d|x| d \Omega(x /|x|) & =\frac{1}{4} \int_{\mathcal{S}^{3}} T_{m}\left((x /|x|)^{-1}\right) A T_{m}(x /|x|) d \Omega(x /|x|) \\
& =\frac{\pi^{2} / 2}{m+1} \operatorname{Tr}(A) .
\end{aligned}
$$

Clearing the denominator of 4 leads to the desired formula for (i) on $\mathcal{S}^{3}$. Now (ii) on $\mathcal{S}^{3}$ follows by exactly the same reasoning. Since $|x|^{-2 m}=1$ on $\mathcal{S}^{3}$, the orthogonality of $t_{i, j}^{m}$ on $\mathcal{S}^{3}$ follows, as does their independence since none of these functions are zero (or have norm zero on $\mathcal{S}^{3}$ ). 
Lemma 2.6(iii) implies that the coefficient functions are linearly independent in both $L^{2}\left(\mathcal{S}^{3}\right)$ and $C\left(\mathcal{R}^{4}\right)$. Indeed, they form a basis for homogeneous harmonic polynomials on $\mathcal{R}^{4}$ and for the spherical harmonics of degree $m$ on $\mathcal{S}^{3}$ (see (3.14)). Hence, for any $p \in \mathcal{N},\left\{t_{i, j}^{m}: 0 \leq i, j \leq m, 0 \leq\right.$ $m \leq p\}$ is linearly independent both on $\mathcal{S}^{3}$ and on $\mathcal{R}^{4}$.

Given the north pole $[1,0]$ and some general vector $x=[z, w]$, we can find a rotation that leaves the north pole fixed and rotates $x$ to a vector in the direction $\left[e^{\mathrm{i} \theta}, 0\right]$ where $\cos \theta=\Re(z) /|x|$. Note that $\theta$ is just the angle between $x$ and the north pole. We could equivalently rotate to a vector in the direction $\left[e^{-\mathbf{i} \theta}, 0\right]$. Hence any function independent of rotation about the north pole must be a function of $|x|$ and $\theta$ and furthermore must be even in $\theta$, i.e., is a function of $\cos \theta$. It is known that all rotations leaving the north pole fixed can be achieved by conjugation, $x \mapsto v x v^{-1}$, with elements of $S U(2)$. See [14, pp. 277-279] or [2, pp. 214-217] for a geometric proof. The same result may be obtained algebraically by considering the diagonalizability of $x$ (see, e.g., [16, pp. 209-210]). Therefore there is a $v \in S U(2)$ such that

$$
x=v \gamma v^{-1}
$$

where

$$
\gamma=|x| \operatorname{diag}\left(e^{\mathbf{i} \theta}, e^{-\mathbf{i} \theta}\right)
$$

Note that conjugation with $-v$ achieves the same rotation as conjugation with $v$, but this is the only nonuniqueness in identifying conjugations with rotations of the equatorial $\mathcal{S}^{2}$. ically,

These conjugation facts lead to explicit formulae for the traces of these representations. Specif-

$$
\begin{aligned}
\operatorname{Tr}\left(T_{m}(x)\right)=\operatorname{Tr}\left(T_{m}\left(v \gamma v^{-1}\right)\right) & =\operatorname{Tr}\left(T_{m}(v) T_{m}(\gamma) T_{m}\left(v^{-1}\right)\right) \\
& =\operatorname{Tr}\left(T_{m}(v) T_{m}(\gamma) T_{m}(v)^{-1}\right)=\operatorname{Tr}\left(T_{m}(\gamma)\right)
\end{aligned}
$$

Using Lemma 2.3,

$$
t_{i, j}^{m}(\gamma)=t_{i, j}^{m}\left(|x| e^{\mathbf{i} \theta}\right)= \begin{cases}|x|^{m} e^{\mathbf{i}(m-2 j) \theta} & i=j \\ 0 & i \neq j\end{cases}
$$

Hence

$$
\begin{aligned}
\operatorname{Tr}\left(T_{m}(x)\right)=\operatorname{Tr}\left(T_{m}(\gamma)\right) & =\sum_{j=0}^{m} t_{j, j}^{m}(\gamma) \\
& =\sum_{j=0}^{m}|\gamma|^{m} e^{\mathbf{i}(m-2 j) \theta} \\
& =|\gamma|^{m} \frac{e^{\mathbf{i}(m+1) \theta}-e^{-\mathbf{i}(m+1) \theta}}{e^{\mathbf{i} \theta}-e^{-\mathbf{i} \theta}}, \quad \text { if } \theta \neq 0 \\
& =|x|^{m} \frac{\sin (m+1) \theta}{\sin \theta},
\end{aligned}
$$

and interpreting $\sin (m+1) \theta / \sin \theta$ in the conventional fashion (as $m+1)$ at $\theta=0$, the expression is also valid there. As usual the character of the representation is defined to be the function $\chi_{m}: \mathcal{H}_{0}^{1} \rightarrow \mathcal{R}$ given by the trace

$$
\chi_{m}(x):=\operatorname{Tr}\left(T_{m}(x)\right)=|x|^{m} \frac{\sin (m+1) \theta}{\sin \theta} .
$$

In particular $\chi_{0}(x)=1$ and $\chi_{1}(x)=\operatorname{Tr}(x)=2|x| \cos \theta$. These $\chi_{m}$ are homogeneous polynomials of degree $m$ in $z, w, \bar{w}$ and $\bar{z}$ since the entries in $T_{m}(x)$ are. We extend the definition of $\chi_{m}$ to $x=0$ by continuity and define $\chi_{-1}=0$. Note that these $\chi_{m}$ are multiples of the Chebyshev polynomials of the second kind as functions of $t=\cos \theta$. 
Lemma 2.7. For $x \in \mathcal{H}^{1}$ and $m \in \mathcal{N}_{0}$,

$$
\chi_{1}(x) \chi_{m}(x)=\chi_{m+1}(x)+|x|^{2} \chi_{m-1}(x) .
$$

Proof. The result is trivially true when $m=0$ or $x=0$. For $m>0$ and $x \neq 0$

$$
\begin{aligned}
\chi_{1}(x) \chi_{m}(x) & =\frac{|x|^{m+1}}{\sin ^{2} \theta}\{\sin (2 \theta) \sin ((m+1) \theta)\} \\
& =\frac{|x|^{m+1}}{\sin ^{2} \theta}\{2 \sin (\theta) \cos (\theta) \sin ((m+1) \theta)\} \\
& =\frac{|x|^{m+1}}{\sin \theta}\{\sin ((m+2) \theta)+\sin (m \theta)\} \\
& =\chi_{m+1}(x)+|x|^{2} \chi_{m-1}(x) .
\end{aligned}
$$

\subsection{Inner and outer functions.}

We will refer to the functions $t_{i, j}^{m}$ of the previous subsection as the inner functions as they will be shown to be homogeneous of non-negative degree and harmonic in $\mathcal{R}^{4}$. The purpose of this subsection is to introduce the outer functions $o_{i, j}^{m}$ which will be shown to be homogeneous of negative degree and harmonic in $\mathcal{R}^{4} \backslash\{0\}$. The subsection also contains the addition formula connecting the inner and outer functions with the character functions.

The representations $T_{m}$ may be used to construct anti-representations, $O_{m}$ of $\mathcal{H}_{0}^{1}$, defined by

$$
O_{m}(x):=|x|^{-2} T_{m}\left(x^{-1}\right)
$$

or, in terms of the coefficient functions,

$$
\begin{aligned}
o_{i, j}^{m}(z, w)=|x|^{-2} t_{i, j}^{m}\left(x^{-1}\right) & =(z \bar{z}+w \bar{w})^{-1} t_{i, j}^{m}\left(\bar{z} /|x|^{2},-w /|x|^{2}\right) \\
& =|x|^{-(2 m+2)} t_{i, j}^{m}(\bar{z},-w) \\
& =|x|^{-2(m+1)} \overline{t_{j, i}^{m}(z, w)}\left(\begin{array}{c}
m \\
i
\end{array}\right)\left(\begin{array}{c}
m \\
j
\end{array}\right)^{-1} .
\end{aligned}
$$

Thus the coefficient functions for $O_{m}$ are homogeneous of degree $-(m+2)$. Together, (2.4) and (2.12) give the equivalent definition of the outer functions

$$
|x|^{2(m+1)} \sum_{i=0}^{m} o_{i, j}^{m}(z, w) z_{1}^{m-i} z_{2}^{i}=\left(z_{1} \bar{z}+z_{2} \bar{w}\right)^{m-j}\left(z_{1}(-w)+z_{2} z\right)^{j} .
$$

See appendix A for tables of low degree inner and outer functions. The operators $O_{m}$ are antirepresentations as

$$
O_{m}(x \cdot y)=O_{m}(y) \cdot O_{m}(x) .
$$

Remark 2.8. When we prove harmonicity of the inner functions, or of the outer functions, the harmonicity of the other set will follow. Indeed definition (2.12) corresponds to an inversion of the functions $t_{i, j}^{m}$ in the unit sphere followed by scaling by $|x|^{-2}$, along with reflection in the $\Re(z)$ axis. This reflection $(z, w) \rightarrow(\bar{z},-w)$ corresponds to quaternionic conjugation. Both this scaled inversion, sometimes called the Kelvin transformation, and the reflection preserve harmonicity.

Associated with the spherical harmonics in $\mathcal{S}^{d-1}$ for any integer $d \geq 2$ is an addition formula [15, pp. 3-10]. These addition formulae express the character function (sometimes called the Gengenbauer polynomial or the Legendre function) at the inner product of two points $u$ and $v$ on $\mathcal{S}^{d-1}$ as a sum of products, in each of which the influence of $u$ and $v$ is separated. Perhaps the best known example of this phenomena is the addition formula for the ordinary Legendre polynomial, $P_{n}(\cos \gamma)$, which is exploited in the multipole expansion of the 3D potential, (see, e.g., $[3,7,8]$ ). With our definition of the inner and outer functions, the addition formula for $\mathcal{R}^{4}$ takes the forms 
Lemma 2.9 (Addition formulae for $\chi_{m}$ ).

(i) If $x, x_{<} \in \mathcal{H}^{1}, x \neq 0$ then

$$
\chi_{m}\left(x^{-1} x_{<}\right)=|x|^{2} \operatorname{Tr}\left(O_{m}(x) T_{m}\left(x_{<}\right)\right)=|x|^{2} \sum_{i, j=0}^{m} t_{j, i}^{m}\left(x_{<}\right) o_{i, j}^{m}(x) .
$$

(ii) If $x, x_{<} \in \mathcal{H}^{1}$ then

$$
\chi_{m}\left(x^{*} x_{<}\right)=\operatorname{Tr}\left(T_{m}\left(x_{<}^{*}\right) T_{m}(x)\right)=\sum_{i, j=0}^{m} t_{j, i}^{m}\left(x_{<}^{*}\right) t_{i, j}^{m}(x) .
$$

Proof. From the definition of the character functions $\chi_{m}$,

$$
\begin{aligned}
\chi_{m}\left(x^{-1} x_{<}\right)=\operatorname{Tr}\left(T_{m}\left(x^{-1} x_{<}\right)\right) & =\operatorname{Tr}\left(T_{m}\left(x^{-1}\right) T_{m}\left(x_{<}\right)\right) \\
& =\sum_{i, j=0}^{m} t_{i, j}^{m}\left(x^{-1}\right) t_{j, i}^{m}\left(x_{<}\right) \\
& =|x|^{2} \sum_{i, j=0}^{m}|x|^{-2} t_{i, j}^{m}\left(x^{-1}\right) t_{j, i}^{m}\left(x_{<}\right) \\
& =|x|^{2} \sum_{i, j=0}^{m} o_{i, j}^{m}(x) t_{j, i}^{m}\left(x_{<}\right),
\end{aligned}
$$

which proves part (i). For part (ii), notice that by (2.9),

$$
\chi_{m}\left(x^{*}\right)=\chi_{m}(x) \text {. }
$$

Therefore

$$
\chi_{m}\left(x^{*} x_{<}\right)=\chi_{m}\left(x_{<}{ }^{*} x\right)=\operatorname{Tr}\left(T_{m}\left(x_{<}{ }^{*}\right) T_{m}(x)\right)=\sum_{i, j=0}^{m} t_{j, i}^{m}\left(x_{<}{ }^{*}\right) t_{i, j}^{m}(x)
$$

The addition formula (2.14) essentially displays the fact that

$$
\frac{m+1}{\pi^{2} / 2} \chi_{m}\left(x^{-1} x_{<}\right)
$$

is a reproducing kernel for $\operatorname{span}\left\{t_{i, j}^{m}, i, j=0, \ldots, m\right\}$, the space of homogeneous harmonic polynomials of degree $m$. In fact the biorthogonality in Lemma 2.6(ii) immediately shows that for any

$$
f_{m}=\sum_{i, j=0}^{m} a_{i, j} t_{i, j}^{m}
$$

in this span,

$$
\int_{0<|x| \leq 1} f_{m}(x) \frac{m+1}{\pi^{2} / 2} \chi_{m}\left(x^{-1} x_{<}\right) d x=f_{m}\left(x_{<}\right) .
$$

\section{Properties of the inner and outer functions.}

Some fundamental properties of the inner and outer functions will be developed in this section. These properties are needed for the development of a fast multipole like method, but are also of interest in their own right. Properties which are considered below include symmetries, recurrence relations, derivative formulae and harmonicity. 


\subsection{Symmetries.}

In this subsection we will develop some symmetry properties of the inner and outer functions. We have already seen an example of a symmetry relation in Lemma 2.5. One application of these symmetry properties is an approximate halving of the costs of forming and evaluating the truncated far field expansions to be developed in Section 4.

Will we use the symbols $\mathrm{i}, \mathrm{j}$ and $\mathrm{k}$ for the fundamental quaternionic units and $\mathrm{i}$ for the imaginary number $\sqrt{-1}$. It will be convenient to use the $2 \times 2$ matrix realisation of the quaternions. In this realisation the quaternion 1 is the $2 \times 2$ identity matrix and

$$
\mathrm{i}=\left[\begin{array}{cc}
\mathbf{i} & 0 \\
0 & -\mathrm{i}
\end{array}\right], \quad \mathrm{j}=\left[\begin{array}{cc}
0 & 1 \\
-1 & 0
\end{array}\right], \quad \mathrm{k}=\left[\begin{array}{cc}
0 & \mathbf{i} \\
-\mathbf{i} & 0
\end{array}\right] .
$$

Now consider conjugation of an element in $\mathcal{H}^{1}$ by $\mathrm{i}$.

$$
\left[\begin{array}{cc}
\mathbf{i} & 0 \\
0 & -\mathbf{i}
\end{array}\right]\left[\begin{array}{cc}
z & w \\
-\bar{w} & \bar{z}
\end{array}\right]\left[\begin{array}{cc}
-\mathbf{i} & 0 \\
0 & \mathbf{i}
\end{array}\right]=\left[\begin{array}{cc}
z & -w \\
\bar{w} & \bar{z}
\end{array}\right] .
$$

Applying $T_{m}$

$$
T_{m}(\mathbf{i}, 0) T_{m}(z, w) T_{m}(-\mathbf{i}, 0)=T_{m}(z,-w) .
$$

By Lemma $2.3 T_{m}(\mathbf{i}, 0)$ and $T_{m}(-\mathbf{i}, 0)$ are diagonal with

$$
t_{i, i}^{m}(\mathbf{i}, 0)=(-1)^{i} \mathbf{i}^{m}, \quad t_{j, j}^{m}(-\mathbf{i}, 0)=(-1)^{m-j} \mathbf{i}^{m},
$$

we conclude that

$$
(-1)^{i} \mathbf{i}^{m} t_{i, j}^{m}(z, w)(-1)^{m-j_{i} m}=t_{i, j}^{m}(z,-w)
$$

for $0 \leq i, j \leq m$. That is

$$
t_{i, j}^{m}(z, w)=(-1)^{i-j} t_{i, j}^{m n}(z,-w)
$$

for $0 \leq i, j \leq m$.

Similar results can be obtain in the same way by conjugation with $j$ and $k$. These results are summarised in the following lemma.

Lemma 3.1. The inner functions $t_{i, j}^{m}$ defined by (2.4) satisfy

$$
\begin{aligned}
& t_{i, j}^{m}(z, w)=(-1)^{i-j} t_{i, j}^{m}(z,-w), \\
& t_{i, j}^{m}(z, w)=(-1)^{i+j} t_{m-i, m-j}^{m}(\bar{z}, \bar{w}), \\
& t_{i, j}^{m}(z, w)=t_{m-i, m-j}^{m}(\bar{z},-\bar{w}),
\end{aligned}
$$

for all $x=[z, w] \in \mathcal{C}^{2}$.

Because each $t_{i, j}^{m}(z, w)$ is a polynomial with real coefficients in $z, w, \bar{z}$ and $\bar{w}$,

$$
\overline{T_{m}(z, w)}=T_{m}(\bar{z}, \bar{w})
$$

Hence $(3.2 b)$ may be written

$$
t_{i, j}^{m}(z, w)=(-1)^{i+j} \overline{t_{m-i, m-j}^{m}(z, w)} .
$$

Using the expression (2.12) for the outer functions in terms of the inner functions each symmetry of $T_{m}$ implies a symmetry of $O_{m}$. In particular, symmetry (3.3) implies

$$
\begin{aligned}
o_{i, j}^{m}(z, w) & =|x|^{-(2 m+2)} t_{i, j}^{m}(\bar{z},-w) \\
& =|x|^{-(2 m+2)}(-1)^{i+j} t_{m-i, m-j}^{m}(z,-\bar{w}) \\
& =(-1)^{i+j} \frac{o_{m-i, m-j}^{m}(z, w)}{m}
\end{aligned}
$$


From symmetries (3.3) and (3.4)

$$
o_{i, j}^{m}(x) t_{j, i}^{m}\left(x_{<}\right)=\overline{o_{m-i, m-j}^{m}(x) t_{m-j, m-i}^{m}\left(x_{<}\right)}
$$

for $0 \leq i, j \leq m$. Hence the terms in the addition formula expression (2.14) for $\chi_{m}\left(x^{-1} x_{<}\right)$are conjugate symmetric with respect to reflection in the middle index $\left(\frac{m}{2}, \frac{m}{2}\right)$. Thus the addition formula can be rewritten to involve a real part and approximately half the number of terms. For example, one such recasting is

$$
\begin{aligned}
\chi_{m}\left(x^{-1} x_{<}\right)= & |x|^{2} \sum_{i, j=0}^{m} o_{i, j}^{m}(x) t_{j, i}^{m}\left(x_{<}\right) \\
& =|x|^{2} \Re\left(\sum_{k=0}^{\left\lfloor\frac{1}{2}\left((m+1)^{2}-1\right)\right\rfloor}\left(2-\delta_{m / 2, i(k)} \delta_{m / 2, j(k)}\right) o_{i(k), j(k)}^{m}(x) t_{j(k), i(k)}^{m}\left(x_{<}\right)\right),
\end{aligned}
$$

where $i(k)=k \bmod (m+1)$ and $j(k)=\lfloor k /(m+1)\rfloor$. This observation can and should be used to halve the storage requirements and flop counts of fast evaluators built upon the analysis of this paper. For example, the recast expression (3.5) can be substituted almost directly into the truncated far field expansion, $g_{p}$, for a sum of shifts of the potential of Theorem 4.2. This will then approximately halve the marginal operation count for evaluating $g_{p}(x)$ at a single extra $x$.

A further symmetry will be useful to recast products of powers of $|x|$ with outer functions as inner functions.

Lemma 3.2. For $m \in \mathcal{N}$ and $0 \leq i, j \leq m$

$$
\left(\begin{array}{c}
m \\
j
\end{array}\right)|x|^{2 m+2} o_{i, j}^{m}(x)=(-1)^{i-j}\left(\begin{array}{c}
m \\
i
\end{array}\right) t_{m-j, m-i}^{m}(x) .
$$

Proof. Substitution of (3.3) into (2.12) gives the result.

\subsection{Recurrence relationships.}

In this subsection we develop recurrence relations which provide efficient methods for calculating the inner and outer functions. For a given $x$ they allow calculation of $\left\{t_{i, j}^{m}, 0 \leq i, j \leq m, 0 \leq m \leq\right.$ $p\}$ or $\left\{o_{i, j}^{m}, 0 \leq i, j \leq m, 0 \leq m \leq p\right\}$ in $\mathcal{O}\left(p^{3}\right)$ operations, which is the same magnitude as the number of terms to be calculated.

It is convenient to extend the definitions of $t_{i, j}^{m}$ and $o_{i, j}^{m}$ by defining $t_{i, j}^{m}=0=o_{i, j}^{m}$ for $i$ or $j \notin[0, m]$.

Lemma 3.3. The inner functions $t_{i, j}^{m}$ defined by (2.4), satisfy the overlapping recurrence relations:

$$
t_{i, j}^{m+1}(z, w)= \begin{cases}z t_{i, j}^{m}(z, w)-\bar{w} t_{i-1, j}^{m}(z, w), & 0 \leq j \leq m, \\ w t_{i, j-1}^{m}(z, w)+\bar{z} t_{i-1, j-1}^{m}(z, w), & 1 \leq j \leq m+1,\end{cases}
$$

for $0 \leq i \leq m+1$, and also satisfy the backward recurrence relations:

$$
\begin{aligned}
|x|^{2} t_{i, j}^{m}(z, w) & =\bar{z} t_{i, j}^{m+1}(z, w)+\bar{w} t_{i, j+1}^{m+1}(z, w) \\
& =-w t_{i+1, j}^{m+1}(z, w)+z t_{i+1, j+1}^{m+1}(z, w)
\end{aligned}
$$

for $0 \leq i, j \leq m$.

Proof. From (2.4), for $1 \leq j \leq m+1$, we obtain

$$
\sum_{i=0}^{m+1} t_{i, j}^{m+1}(z, w) z_{1}^{m+1-i} z_{2}^{i}=\left(z z_{1}-\bar{w} z_{2}\right)^{m+1-j}\left(w z_{1}+\bar{z} z_{2}\right)^{j}
$$




$$
\begin{aligned}
& =\left\{\left(z z_{1}-\bar{w} z_{2}\right)^{m-(j-1)}\left(w z_{1}+\bar{z} z_{2}\right)^{j-1}\right\}\left(w z_{1}+\bar{z} z_{2}\right) \\
& =\left\{\sum_{i=0}^{m} t_{i, j-1}^{m}(z, w) z_{1}^{m-i} z_{2}^{i}\right\}\left(w z_{1}+\bar{z} z_{2}\right) \\
& =\sum_{i=0}^{m} t_{i, j-1}^{m}(z, w)\left\{w z_{1}^{m+1-i} z_{2}^{i}+\bar{z} z_{1}^{m-i} z_{2}^{i+1}\right\} \\
& =\sum_{i=0}^{m} t_{i, j-1}^{m}(z, w) w z_{1}^{m+1-i} z_{2}^{i}+\sum_{i=0}^{m} t_{i, j-1}^{m}(z, w) \bar{z} z_{1}^{(m+1)-(i+1)} z_{2}^{i+1} \\
& =\sum_{i=0}^{m} w t_{i, j-1}^{m}(z, w) z_{1}^{m+1-i} z_{2}^{i}+\sum_{i=1}^{m+1} \bar{z} t_{i-1, j-1}^{m}(z, w) z_{1}^{m+1-i} z_{2}^{i} \\
& =\sum_{i=0}^{m+1}\left\{w t_{i, j-1}^{m}(z, w)+\bar{z} t_{i-1, j-1}^{m}(z, w)\right\} z_{1}^{m+1-i} z_{2}^{i} .
\end{aligned}
$$

Equating coefficients of $z_{1}^{m+1-i} z_{2}^{i}$ gives the second part of (3.6). The first part may be obtained in a similar manner, or more directly by application of the symmetry relation (3.2b) to both sides of the part just proved.

The backward recursion may be obtained directly from the forward recursion. Multiplying the first right-hand side of (3.6) by $\bar{z}$ and the second right-hand side (with $j$ replaced by $j+1$ ) by $\bar{w}$ and summing gives the first right-hand side of (3.7). The second right-hand side is similar.

These formulae are analogous to the addition formulae which express $\cos (m \pm 1) \theta$ and $\sin (m \pm$ 1) $\theta$ in terms of $\cos \theta, \sin \theta, \sin m \theta$ and $\cos m \theta$.

Lemma 3.4. The outer functions $o_{i, j}^{m}$ defined by (2.12), satisfy the overlapping recurrence relations:

$$
o_{i, j}^{m+1}(z, w)= \begin{cases}\frac{\bar{z}}{|x|^{2}} o_{i, j}^{m}(z, w)+\frac{\bar{w}}{|x|^{2}} o_{i-1, j}^{m}(z, w), & 0 \leq j \leq m, \\ \frac{-w}{|x|^{2}} o_{i, j-1}^{m}(z, w)+\frac{z}{|x|^{2}} o_{i-1, j-1}^{m}(z, w), & 1 \leq j \leq m+1,\end{cases}
$$

for $0 \leq i \leq m+1$, and also satisfy the backward recurrence relations:

$$
\begin{aligned}
o_{i, j}^{m}(z, w) & =z o_{i, j}^{m+1}(z, w)-\bar{w} o_{i, j+1}^{m+1}(z, w) \\
& =w o_{i+1, j}^{m+1}(z, w)+\bar{z} o_{i+1, j+1}^{m+1}(z, w)
\end{aligned}
$$

for $0 \leq i, j \leq m$.

Proof. This follows easily by substituting the expression (2.12) for $o_{i, j}^{m}$ into the relations (3.6) and (3.7) for the inner functions $t_{i, j}^{m}$.

\subsection{Derivatives and harmonicity.}

In this subsection we develop derivative formulae for the inner and outer functions. Applications include proofs of harmonicity, a dual basis for the inner functions, and an expression for the outer functions as an appropriate derivative of $1 /|x|^{2}$. Analogous formulae in the three dimensional case are given in Epton and Dembart [8].

We start by recalling the definitions for the complex derivative operators:

$$
\begin{aligned}
\frac{\partial}{\partial z} & =\frac{1}{2}\left(\frac{\partial}{\partial x_{1}}-\mathbf{i} \frac{\partial}{\partial x_{2}}\right), & \frac{\partial}{\partial \bar{z}} & =\frac{1}{2}\left(\frac{\partial}{\partial x_{1}}+\mathbf{i} \frac{\partial}{\partial x_{2}}\right), \\
\frac{\partial}{\partial w} & =\frac{1}{2}\left(\frac{\partial}{\partial x_{3}}-\mathrm{i} \frac{\partial}{\partial x_{4}}\right), & \frac{\partial}{\partial \bar{w}} & =\frac{1}{2}\left(\frac{\partial}{\partial x_{3}}+\mathrm{i} \frac{\partial}{\partial x_{4}}\right) .
\end{aligned}
$$


By considering $z, \bar{z}, w$ and $\bar{w}$ as functions of $x_{1}, x_{2}, x_{3}$ and $x_{4}$, these operators may be applied to functions defined on $\mathcal{C}^{2}$ in the natural way. Indeed, immediate consequences of these definitions are the relations

$$
\frac{\partial}{\partial z} \bar{z}=0, \quad \text { and } \quad \frac{\partial}{\partial z} z=1,
$$

their conjugates and the similar relations with $w, \bar{w}$. More generally, if $f(x)=h(z, \bar{z}, w, \bar{w})$ is complex analytic in $z$, i.e., independent of $\bar{z}$ in the sense that $\partial h / \partial \bar{z}=0$, then $\partial f / \partial z$ is given by all the usual rules for differentiation. Furthermore, armed with these operators the ordinary Laplacian may be expressed as

$$
\Delta=4\left(\frac{\partial^{2}}{\partial z \partial \bar{z}}+\frac{\partial^{2}}{\partial w \partial \bar{w}}\right) \cdot
$$

Lemma 3.5. Any of the first partial derivatives map the inner functions of degree $m$ to (multiples of) inner functions of degree $m-1$. Specifically, for $0 \leq i, j \leq m+1$,

$$
\begin{aligned}
\frac{\partial}{\partial z} t_{i, j}^{m+1}(z, w) & =(m+1-j) t_{i, j}^{m}(z, w) \\
\frac{\partial}{\partial \bar{w}} t_{i, j}^{m+1}(z, w) & =-(m+1-j) t_{i-1, j}^{m}(z, w) \\
\frac{\partial}{\partial w} t_{i, j}^{m+1}(z, w) & =j t_{i, j-1}^{m}(z, w) \\
\frac{\partial}{\partial \bar{z}} t_{i, j}^{m+1}(z, w) & =j t_{i-1, j-1}^{m}(z, w)
\end{aligned}
$$

Proof. Differentiating (2.4) with respect to $z$ gives

$$
\begin{aligned}
\sum_{i=0}^{m+1} \frac{\partial}{\partial z} t_{i, j}^{m+1}(z, w) z_{1}^{m+1-i} z_{2}^{i} & =(m+1-j) z_{1}\left(z_{1} z-z_{2} \bar{w}\right)^{m-j}\left(z_{1} w+z_{2} \bar{z}\right)^{j} \\
& =(m+1-j) z_{1} \sum_{i=0}^{m} t_{i, j}^{m}(z, w) z_{1}^{m-i} z_{2}^{i} \\
& =(m+1-j) \sum_{i=0}^{m} t_{i, j}^{m}(z, w) z_{1}^{m+1-i} z_{2}^{i} .
\end{aligned}
$$

Equating coefficients gives (3.12a). In a similar manner, by differentiating (2.4) with respect to $\bar{w}, w$ and $\bar{z}$ we may obtain $(3.12 \mathrm{~b}),(3.12 \mathrm{c})$ and (3.12d) respectively.

Lemma 3.6. Any of the first partial derivatives map the outer functions of degree $-m-2$ to (multiples of) outer functions of degree $-m-3$. Specifically, for $m \geq 0$ and $0 \leq j \leq m$,

$$
\begin{aligned}
\frac{\partial}{\partial z} o_{i, j}^{m}(z, w) & =-(m+1-i) o_{i, j}^{m+1}(z, w), & & 0 \leq i \leq m+1, \\
\frac{\partial}{\partial \bar{w}} o_{i, j}^{m}(z, w) & =(m+1-i) o_{i, j+1}^{m+1}(z, w), & & \leq \leq i \leq m+1, \\
\frac{\partial}{\partial w} o_{i, j}^{m}(z, w) & =-(i+1) o_{i+1, j}^{m+1}(z, w), & -1 & \leq i \leq m, \\
\frac{\partial}{\partial \bar{z}} o_{i, j}^{m}(z, w) & =-(i+1) o_{i+1, j+1}^{m+1}(z, w), & -1 & \leq i \leq m .
\end{aligned}
$$

Proof of (3.13a). The proof is by induction on $m$.

Induction basis. Since

$$
o_{0,0}^{0}(z, w)=(z \bar{z}+w \bar{w})^{-1},
$$

an application of (3.8) shows

$$
O_{1}(z, w)=|x|^{-4}\left[\begin{array}{cc}
\bar{z} & -w \\
\bar{w} & z
\end{array}\right]
$$


It follows that (3.13a) is true for $m=0$.

Induction step. Assume that (3.13a) is true for $0 \leq m \leq M$. By the first part of (3.8),

$$
o_{i, j}^{M+1}(z, w)=\frac{\bar{z}}{|x|^{2}} o_{i, j}^{M}(z, w)+\frac{\bar{w}}{|x|^{2}} o_{i-1, j}^{M}(z, w),
$$

for $0 \leq i \leq M+1$ and $0 \leq j \leq M$. Using the inductive hypothesis to differentiate the outer functions,

$$
\begin{aligned}
\frac{\partial}{\partial z} o_{i, j}^{M+1}(x)= & \frac{\bar{z}(-\bar{z})}{|x|^{4}} o_{i, j}^{M}(x)+\frac{\bar{z}}{|x|^{2}} \frac{\partial}{\partial z} o_{i, j}^{M}(x)+\frac{\bar{w}(-\bar{z})}{|x|^{4}} o_{i-1, j}^{M}(x)+\frac{\bar{w}}{|x|^{2}} \frac{\partial}{\partial z} o_{i-1, j}^{M}(x) \\
= & \frac{-\bar{z}}{|x|^{2}}\left(\frac{\bar{z}}{|x|^{2}} o_{i, j}^{M}(x)+\frac{\overline{\bar{w}}}{|x|^{2}} o_{i-1, j}^{M}(x)\right)-(M+1-i) \frac{\bar{z}}{|x|^{2}} o_{i, j}^{M+1}(x) \\
& -(M+2-i) \frac{\bar{w}}{|x|^{2}} o_{i-1, j}^{M+1}(x) .
\end{aligned}
$$

Two applications of the first part of (3.8) give the result.

However, if $j=M+1$ the above does not hold. By the second part of (3.8),

$$
o_{i, j}^{M+1}(z, w)=\frac{-w}{|x|^{2}} o_{i, j-1}^{M}(z, w)+\frac{z}{|x|^{2}} o_{i-1, j-1}^{M}(z, w),
$$

for $0 \leq i \leq M+1$ and $1 \leq j \leq M+1$. Once again the inductive hypothesis will be used to differentiate this expression. This gives

$$
\begin{aligned}
\frac{\partial}{\partial z} o_{i, j}^{M+1}(x)= & \frac{(-w)(-\bar{z})}{|x|^{4}} o_{i, j-1}^{M}(x)+\frac{(-w)}{|x|^{2}} \frac{\partial}{\partial z} o_{i, j-1}^{M}(x) \\
& +\frac{z(-\bar{z})}{|x|^{4}} o_{i-1, j-1}^{M}(x)+\frac{z}{|x|^{2}} \frac{\partial}{\partial z} o_{i-1, j-1}^{M}(x)+\frac{1}{|x|^{2}} o_{i-1, j-1}^{M}(x) \\
= & \frac{(-w)(-\bar{z})}{|x|^{4}} o_{i, j-1}^{M}(x)-(M+1-i) \frac{(-w)}{|x|^{2}} o_{i, j-1}^{M+1}(x) \\
& +\frac{z(-\bar{z})}{|x|^{4}} o_{i-1, j-1}^{M}(x)-(M+2-i) \frac{z}{|x|^{2}} o_{i-1, j-1}^{M+1}(x)+\frac{z \bar{z}+w \bar{w}}{|x|^{4}} o_{i-1, j-1}^{M}(x) \\
= & -(M+1-i) \frac{(-w)}{|x|^{2}} o_{i, j-1}^{M+1}(x)-(M+2-i) \frac{z}{|x|^{2}} o_{i-1, j-1}^{M+1}(x) \\
& -\frac{(-w)}{|x|^{2}}\left(\frac{\bar{z}}{|x|^{2}} o_{i, j-1}^{M}(x)+\frac{\bar{w}}{|x|^{2}} o_{i-1, j-1}^{M}(x)\right) .
\end{aligned}
$$

By the first part of (3.8), the term in the brackets is equal to $o_{i, j-1}^{M+1}(z, w)$ and thus the second part of (3.8) gives the required result. This proves (3.13a) by induction on $m$. The other three relations in (3.13) may be proven in a similar way.

Given the above expressions for the derivatives of the inner and outer functions it is easy to show that they are harmonic functions. Since (3.11) may be used for the Laplacian, it follows that

$$
\frac{1}{4} \Delta o_{i, j}^{m}=(-(m+1-i))(-(i+1)) o_{i+1, j+1}^{m+2}+(m+1-i)(-(i+1)) o_{i+1, j+1}^{m+2}=0
$$

and

$$
\frac{1}{4} \Delta t_{i, j}^{m+2}=j(m+2-j) t_{i-1, j-1}^{m}-j(m+2-j) t_{i-1, j-1}^{m}=0 .
$$

Each of (3.14) and (3.15) can be inferred from the other as indicated in Remark 2.8.

From the product rule for the Laplacian,

$$
\Delta(f g)=(\Delta f) g+2(\nabla f) \cdot(\nabla g)+f(\Delta g)
$$


and the Euler relation for a function $f$ that is homogeneous of degree $m$,

$$
x \cdot(\nabla f)(x)=m f(x)
$$

we easily obtain

Lemma 3.7. Let $|\cdot|$ be the 2-norm on $\mathcal{R}^{d}$. If $f: \mathcal{R}^{d} \rightarrow \mathcal{R}$ is a harmonic function that is homogeneous of degree $m$ then

$$
\Delta\left(|\cdot|^{2 \ell} f\right)=4 \ell\left(\frac{d}{2}+\ell+m-1\right)|\cdot|^{2(\ell-1)} f
$$

and hence $|\cdot|{ }^{2 \ell} f$ is polyharmonic of order $(\ell+1)$.

\section{Corollary 3.8.}

$$
\begin{aligned}
& \Delta\left(|\cdot|{ }^{2 \ell} o_{i, j}^{m}\right)=4 \ell(\ell-m-1)|\cdot|^{2(\ell-1)} o_{i, j}^{m}, \\
& \Delta\left(|\cdot|{ }^{2 \ell} t_{i, j}^{m}\right)=4 \ell(\ell+m+1)|\cdot|^{2(\ell-1)} t_{i, j}^{m} .
\end{aligned}
$$

All of the outer functions $o_{i, j}^{m}(x)$ may be written as (multiples of) derivatives of $1 /|x|^{2}$ as in the following.

Lemma 3.9. Define

$$
R_{i, j}^{m}= \begin{cases}\frac{(-1)^{m-j+i}}{i !(m-i) !} \frac{\partial^{m}}{\partial \bar{w}^{j-i} \partial z^{m-j} \partial \bar{z}^{i}}, & i \leq j \\ \frac{(-1)^{m}}{i !(m-i) !} \frac{\partial^{m}}{\partial w^{i-j} \partial z^{m-i} \partial \bar{z}^{j}}, & i \geq j\end{cases}
$$

$0 \leq i, j \leq m, m \in \mathcal{N}_{0}$. Then for $x \in \mathcal{R}^{4} \backslash\{0\}$,

$$
o_{i, j}^{m}(x)=R_{i, j}^{m} \frac{1}{|x|^{2}} .
$$

Furthermore, for harmonic functions $f$,

$$
R_{i, j}^{m} R_{i^{\prime}, j^{\prime}}^{m^{\prime}} f=e\left(m, m^{\prime}, i\right)_{i^{\prime}, i^{\prime}} R_{i+i^{\prime}, j+j^{\prime}}^{m+m^{\prime}} f
$$

where

$$
e\left(m, m^{\prime}, i\right)_{i^{\prime}, i^{\prime}}=\left(\begin{array}{c}
i+i^{\prime} \\
i
\end{array}\right)\left(\begin{array}{c}
m+m^{\prime}-\left(i+i^{\prime}\right) \\
m-i
\end{array}\right) .
$$

Proof. The definition of the outer functions in terms of the inner functions, (2.12), and the fact that $t_{0,0}^{0}=1$, imply

$$
o_{0,0}^{0}(x)=\frac{1}{|x|^{2}} .
$$

Then (3.17) follows by repeated use of Lemma 3.6.

Consider now (3.18). If $i \leq j$ and $i^{\prime} \leq j^{\prime}$ or $i \geq j$ and $i^{\prime} \geq j^{\prime}$, then (3.18) follows easily from (3.16). Turning to the slightly more difficult mixed case assume, without loss of generality, $i \leq j$ and $i^{\prime} \geq j^{\prime}$. Using Definition (3.16),

$$
R_{i, j}^{m} R_{i^{\prime}, j^{\prime}}^{m^{\prime}} f=\frac{(-1)^{m-j+i+m^{\prime}}}{i !(m-i) ! i^{\prime} !\left(m^{\prime}-i^{\prime}\right) !} \frac{\partial^{m+m^{\prime}}}{\partial \bar{w}^{j-i} \partial w^{i^{\prime}}-j^{\prime} \partial z^{m-j+m^{\prime}-i^{\prime}} \partial \bar{z}^{i+j^{\prime}}} f .
$$

Let $g$ be harmonic. Then from the form (3.11) for the Laplacian

$$
\frac{\partial^{2}}{\partial z \partial \bar{z}} g=-\frac{\partial^{2}}{\partial w \partial \bar{w}} g
$$


Hence, if $i^{\prime}-j^{\prime} \geq j-i$, then

$$
\frac{\partial^{i^{\prime}-j^{\prime}+j-i}}{\partial \bar{w}^{j-i} \partial w^{i^{\prime}-j^{\prime}}} f=(-1)^{j-i} \frac{\partial^{i^{\prime}-j^{\prime}+j-i}}{\partial w^{i+i^{\prime}-\left(j+j^{\prime}\right)} \partial z^{j-i} \partial \bar{z}^{j-i}} f .
$$

Substituting this into $(3.20)$ gives

$$
\begin{aligned}
R_{i, j}^{m} R_{i^{\prime}, j^{\prime}}^{m^{\prime}} f & =\frac{(-1)^{m+m^{\prime}}}{i !(m-i) ! i^{\prime} !\left(m^{\prime}-i^{\prime}\right) !} \frac{\partial^{m+m^{\prime}}}{\partial w^{i+i^{\prime}-\left(j+j^{\prime}\right)} \partial z^{m+m^{\prime}-\left(i+i^{\prime}\right)} \partial \bar{z}^{j+j^{\prime}}} f \\
& =e\left(m, m^{\prime}, i\right)_{i^{\prime}, i^{\prime}} R_{i+i^{\prime}, j+j^{\prime}}^{m+m^{\prime}} f
\end{aligned}
$$

since $i^{\prime}-j^{\prime} \geq j-i$ implies $i+i^{\prime} \geq j+j^{\prime}$. The case when $i^{\prime}-j^{\prime} \leq j-i$ is similar.

Motivated by the operators $R_{i, j}^{m}$ we are led to define operators

$$
L_{i, j}^{m}= \begin{cases}\frac{1}{j !(m-j) !} \frac{\partial^{m}}{\partial w^{j-i} \partial \bar{z}^{i} \partial z^{m-j}} & i \leq j \\ \frac{(-1)^{i-j}}{j !(m-j) !} \frac{\partial^{m}}{\partial \bar{w}^{i-j} \partial \bar{z}^{j} \partial z^{m-i}} & i \geq j\end{cases}
$$

Repeated use of Lemma 3.5 then shows

Lemma 3.10 (Dual basis for the inner functions). The operators $L_{i, j}^{m}$ satisfy

$$
L_{i, j}^{m} t_{i, j}^{m}=t_{0,0}^{0},
$$

and also have the more general property

$$
L_{i^{\prime}, j^{\prime}}^{m^{\prime}} t_{i, j}^{m}=\left(\begin{array}{c}
j \\
j^{\prime}
\end{array}\right)\left(\begin{array}{c}
m-j \\
m^{\prime}-j^{\prime}
\end{array}\right) t_{i-i^{\prime}, j-j^{\prime}}^{m-m^{\prime}}
$$

Thus the functionals

$$
\lambda_{i, j}^{m}(f)=\left(L_{i, j}^{m} f\right)(0)
$$

$0 \leq i, j \leq m, 0 \leq m \leq p$, form a dual basis for $\left\{t_{i, j}^{m}, 0 \leq i, j \leq m, 0 \leq m \leq p\right\}$.

\section{Expansions of polyharmonic basic functions.}

In this section we develop far field expansions of the functions $\left|\cdot-x_{<}\right|^{-2}$, and $\left|\cdot-x_{<}\right|^{2 n} \ln \left(\left|\cdot-x_{<}\right|\right)$, $n=0,1, \ldots$. These functions lead to polyharmonic splines of order 1 and $n+2$ respectively. We also find bounds on the error in approximating the associated polyharmonic radial basis functions by truncating these far field expansions. We will truncate by dropping all terms of sufficiently negative homogeneity.

We will find it useful in this section to make use of the cosine formula in the form

$$
\begin{aligned}
\left|x-x_{<}\right|^{2} & =|x|^{2}+\left|x_{<}\right|^{2}-2\left\langle x, x_{<}\right\rangle \\
& =|x|^{2}+\left|x_{<}\right|^{2}-|x|^{2} \operatorname{Tr}\left(x^{-1} x_{<}\right) \\
& =|x|^{2}+\left|x_{<}\right|^{2}-|x|^{2} \chi_{1}\left(x^{-1} x_{<}\right) \\
& =|x|^{2}+\left|x_{<}\right|^{2}-\chi_{1}\left(x^{*} x_{<}\right) \\
& =|x|^{2}+\left|x_{<}\right|^{2}-\chi_{1}\left(x_{<}^{*} x\right) .
\end{aligned}
$$




\subsection{Expansion of the potential.}

We start with a far field expansion of the potential function $\left|x-x_{<}\right|^{-2}$. This is an expansion in the character functions, which are products of powers of $|x<| /|x|$ and the appropriate Gengenbauer polynomials-the Chebyshev polynomials of the second kind.

Lemma 4.1. For $x, x_{<} \in \mathcal{R}^{4}$ with $\left|x_{<}\right|<|x|$,

$$
\frac{1}{\left|x-x_{<}\right|^{2}}=\frac{1}{|x|^{2}} \sum_{m=0}^{\infty} \chi_{m}\left(x^{-1} x_{<}\right) \text {. }
$$

Proof. The result is trivially true when $x_{<}=0$. Hence in what follows we assume that $0<\left|x_{<}\right|<$ $|x|$. Then from the definition of the character function, (2.9),

$$
\chi_{m}\left(x^{-1} x_{<}\right)=\left(\frac{\left|x_{<}\right|}{|x|}\right)^{m} \frac{\sin (m+1) \theta}{\sin \theta}
$$

where $\theta$ is the angle between $x$ and $x_{<}$. As is well known,

$$
\left|\frac{\sin (m+1) \theta}{\sin \theta}\right| \leq m+1, \quad \forall \theta \in \mathcal{R}
$$

Therefore the series on the right of (4.2) converges absolutely.

We will prove the lemma by showing the product of the right hand side of $(4.2)$ with $\left|x-x_{<}\right|^{2}$ is 1 . Let $y=x^{-1} x_{<}$, then

$$
\begin{aligned}
\left|x-x_{<}\right|^{2} \frac{1}{|x|^{2}} \sum_{m=0}^{\infty} \chi_{m}(y) & =\left\{|x|^{2}+\left|x_{<}\right|^{2}-|x|^{2} \chi_{1}(y)\right\} \frac{1}{|x|^{2}} \sum_{m=0}^{\infty} \chi_{m}(y) \\
& =\left\{1+|y|^{2}-\chi_{1}(y)\right\} \sum_{m=0}^{\infty} \chi_{m}(y) \\
& =\sum_{m=0}^{\infty}\left\{\chi_{m}(y)+|y|^{2} \chi_{m}(y)-\chi_{1}(y) \chi_{m}(y)\right\} \\
& =\sum_{m=0}^{\infty}\left\{\chi_{m}(y)+|y|^{2} \chi_{m}(y)-\chi_{m+1}(y)-|y|^{2} \chi_{m-1}(y)\right\} \\
& =\chi_{0}(y)-|y|^{2} \chi_{-1}(y) \\
& =1,
\end{aligned}
$$

where we have used Lemma 2.7 to expand the product $\chi_{1}(y) \chi_{m}(y)$ and the fact that $\chi_{-1}=0$.

We now wish to obtain a bound on the error in approximating $\Phi_{x_{<}}(\cdot)=1 /\left|\cdot-x_{<}\right|^{2}$ by the truncated series

$$
g_{p}(x)=\frac{1}{|x|^{2}} \sum_{m=0}^{p} \chi_{m}\left(x^{-1} x_{<}\right) .
$$

From the explicit formula for the character function, $(2.9),\left|\chi_{m}(y)\right| \leq(m+1)|y|^{m}$ for all $y \in \mathcal{H}^{1}$ and therefore the error in approximating $\Phi_{x_{<}}(\cdot)$ by $g_{p}$ is bounded by

$$
\begin{aligned}
\left|\Phi_{x_{<}}(x)-g_{p}(x)\right| & \leq \frac{1}{|x|^{2}} \sum_{m=p+1}^{\infty}\left|\chi_{m}\left(x^{-1} x_{<}\right)\right| \\
& \leq \frac{1}{|x|^{2}} \sum_{m=p+1}^{\infty}(m+1)|y|^{m}
\end{aligned}
$$




$$
\begin{aligned}
& =\frac{|y|^{p+1}}{|x|^{2}} \sum_{m=0}^{\infty}((p+1)+(m+1))|y|^{m} \\
& =\frac{|y|^{p+1}}{|x|^{2}}\left\{(p+1) \sum_{m=0}^{\infty}|y|^{m}+\sum_{m=0}^{\infty}(m+1)|y|^{m}\right\} .
\end{aligned}
$$

where $y=x^{-1} x_{<}$. If $\left|x_{<}\right|<|x|$ then $|y|<1$ and the well known identities

$$
\sum_{m=0}^{\infty} h^{m}=\frac{1}{1-h} \quad \text { and } \quad \sum_{m=0}^{\infty}(m+1) h^{m}=\frac{1}{(1-h)^{2}}
$$

for $|h|<1$, may be applied. This gives

$$
\left|\Phi_{x_{<}}(x)-g_{p}(x)\right| \leq \frac{|y|^{p+1}}{|x|^{2}}\left\{\frac{p+1}{1-|y|}+\frac{1}{(1-|y|)^{2}}\right\}
$$

Denote the bound on the right of (4.3) by $e_{p}(|y|)$. For $|x|$ fixed, since each term on the right in (4.3) is obviously strictly increasing in $|y|$ for $0<|y|<1$, so is $e_{p}(|y|)$. Considering now the sum

$$
s(x)=\sum_{k=1}^{N} \frac{d_{k}}{\left|x-x_{k}\right|^{2}}
$$

we apply the bound above to each term and sum. The monotonicity of the bound enables us to estimate $e_{p}\left(\left|x_{k}\right| /|x|\right)$ by $e_{p}(d)$ where

$$
d=\max _{1 \leq k \leq N} \frac{\left|x_{k}\right|}{|x|}
$$

In combination with Lemma 4.1 and (2.14), this gives

Theorem 4.2. Suppose $x_{k} \in \mathcal{R}^{4},\left|x_{k}\right| \leq r$ and $d_{k} \in \mathcal{R}$ for each $1 \leq k \leq N$. Let

$$
s(x)=\sum_{k=1}^{N} \frac{d_{k}}{\left|x-x_{k}\right|^{2}}
$$

and let $C_{m}$ be the $(m+1) \times(m+1)$ matrix

$$
\left[C_{i, j}^{m}\right]=\sum_{k=1}^{N} d_{k}\left(T_{m}\left(x_{k}\right)\right)
$$

For $p \in \mathcal{N}_{0}$, set

$$
g_{p}(x)=\sum_{m=0}^{p} \sum_{i, j=0}^{m} C_{j, i}^{m} o_{i, j}^{m}(x)=\sum_{m=0}^{p} \operatorname{Tr}\left(C_{m} O_{m}(x)\right),
$$

$x \in \mathcal{R}^{4} \backslash\{0\}$. Then for all $x$ with $|x|>r$

$$
\left|s(x)-g_{p}(x)\right| \leq \frac{M}{r^{2}}\left(\frac{p+1}{1-1 / c}+\frac{1}{(1-1 / c)^{2}}\right)\left(\frac{1}{c}\right)^{p+3}
$$

where $M=\sum_{k=1}^{N}\left|d_{k}\right|$ and $c=|x| / r$. 


\subsection{Expansion of a polyharmonic function.}

In this subsection our aim is to develop far field expansions for the functions $\left|\cdot-x_{<}\right|^{2 n} \ln \left|\cdot-x_{<}\right|$, which are polyharmonic of order $n+2$. This will be done by induction on $n$ with the biharmonic case $\ln \left|\cdot-x_{<}\right|$being used as the induction basis.

The polyharmonic functions $f$ of order $n+2$ that occur will be written in the form

$$
f=f_{0}+|\cdot|^{2} f_{1}+\cdots+|\cdot|^{2 n+2} f_{n+1}
$$

where $f_{0}, \ldots, f_{n+1}$ are harmonic. In this sum, $|\cdot|{ }^{2 j} f_{j}$ is actually a $(j+1)$-harmonic term. As a consequence the terms of a specified homogeneous order $k$ in our expansions will no longer involve a single $\chi_{m}$ as in the harmonic case of Lemma 4.1. Rather they will be a weighted sum of $\chi_{m}(y)$, $|y|^{2} \chi_{m-2}(y), \ldots,|y|^{2 n+2} \chi_{m-2 n-2}(y)$, consistent with the polyharmonicity orders of Lemma 3.7 . For this reason we need to know how pairs of character functions combine.

Lemma 4.3. For $m \in \mathcal{N}, m \geq 2$ and $\left|x_{<}\right|<|x|$

$$
\chi_{m}\left(x^{-1} x_{<}\right)-\frac{\left|x_{<}\right|^{2}}{|x|^{2}} \chi_{m-2}\left(x^{-1} x_{<}\right)=2 \frac{\left|x_{<}\right|^{m}}{|x|^{m}} \cos (m \theta),
$$

where $\theta$ is the angle between $x$ and $x_{<}$.

Proof. The Lemma is trivially true when $x_{<}=0$. For $0<\left|x_{<}\right|<|x|$, the explicit formula for the character function implies

$$
\begin{aligned}
\chi_{m}\left(x^{-1} x_{<}\right)-\frac{\left|x_{<}\right|^{2}}{|x|^{2}} \chi_{m-2}\left(x^{-1} x_{<}\right) & =\frac{\left|x_{<}\right|^{m}}{|x|^{m}}\left(\frac{\sin (m+1) \theta}{\sin \theta}-\frac{\sin (m-1) \theta}{\sin \theta}\right) \\
& =\frac{\left|x_{<}\right|^{m}}{|x|^{m}}\left(\frac{\sin (m+1) \theta-\sin (m-1) \theta}{\sin \theta}\right) \\
& =2 \frac{\left|x_{<}\right|^{m}}{|x|^{m}} \frac{\sin (\theta) \cos (m \theta)}{\sin \theta} \\
& =2 \frac{\left|x_{<}\right|^{m}}{|x|^{m}} \cos (m \theta) .
\end{aligned}
$$

We now proceed to obtain a far field expansion for the biharmonic function $\ln \left|\cdot-x_{<}\right|$. While this expansion is useful in and of itself, we will also use it as the induction basis for the expansion of the more general function $\left|\cdot-x_{<}\right|^{2 n} \ln \left|\cdot-x_{<}\right|$, which is polyharmonic of order $n+2$.

Lemma 4.4. For $x, x_{<} \in \mathcal{R}^{4}$ and $\left|x_{<}\right|<|x|$,

$$
\ln \left|x-x_{<}\right|^{2}=\ln |x|^{2}-\sum_{m=1}^{\infty} \frac{1}{m}\left\{\chi_{m}\left(x^{-1} x_{<}\right)-\frac{\left|x_{<}\right|^{2}}{|x|^{2}} \chi_{m-2}\left(x^{-1} x_{<}\right)\right\} .
$$

Proof. The case $x_{<}=0$ is trivially true. Hence we assume $0<\left|x_{<}\right|<|x|$.

We will use $\ln (\cdot)$ for the real $\operatorname{logarithm}$ and $\log (\cdot)$ for the principal branch of the complex logarithm. Thus $\Re(\log z)=\ln |z|$ away from the branch cut. We will represent $x=\left(x_{1}, x_{2}, x_{3}, x_{4}\right)^{T} \in$ $\mathcal{R}^{4}$ by $x=\left[x_{1}+\mathrm{i} x_{2}, x_{3}+\mathrm{i} x_{4}\right] \in \mathcal{C}^{2}$ and similarly for $x_{<}$. There exists a rotation $R_{1}$ such that

$$
R_{1} x=[|x|, 0] .
$$

By the argument that precedes the introduction of the character function $\chi_{m}$ in (2.9), there is a rotation $R_{2}$ that fixes the north pole $[1,0]$ and rotates $R_{1} x_{<}$to the direction $\left[e^{\mathbf{i} \theta}, 0\right]$ where $\theta$ is the angle between $R_{1} x_{<}$and the north pole. Since rotations preserve angles $\theta$ is also the angle between $x$ and $x_{<}, R=R_{2} R_{1}$ is a rotation such that

$$
R x=[|x|, 0], \quad R x_{<}=\left[\left|x_{<}\right| e^{\mathbf{i} \theta}, 0\right] .
$$


Thus

$$
\left|x-x_{<}\right|=\left|R\left(x-x_{<}\right)\right|=\left|R x-R x_{<}\right|=\left|\left[|x|-\left|x_{<}\right| e^{\mathrm{i} \theta}, 0\right]\right|=|x|\left|1-\frac{\left|x_{<}\right|}{|x|} e^{\mathrm{i} \theta}\right|=|x||1-| y\left|e^{\mathrm{i} \theta}\right|,
$$

where $y=x^{-1} x_{<}$, and

$$
\begin{aligned}
\ln \left|x-x_{<}\right|=\Re\left\{\log \left(|x|\left(1-|y| e^{\mathrm{i} \theta}\right)\right)\right\} & =\Re\left\{\log (|x|)+\log \left(1-|y| e^{\mathbf{i} \theta}\right)\right\} \\
& =\ln |x|+\Re\left\{\log \left(1-|y| e^{\mathbf{i} \theta}\right)\right\} .
\end{aligned}
$$

Now

$$
\log \left(1-|y| e^{\mathrm{i} \theta}\right)=-\sum_{m=1}^{\infty} \frac{1}{m}\left(|y| e^{\mathrm{i} \theta}\right)^{m}=-\sum_{m=1}^{\infty} \frac{1}{m}|y|^{m} e^{\mathrm{i} m \theta} .
$$

Taking the real part of this expression,

$$
\begin{aligned}
\Re\left\{\log \left(1-\frac{\left|x_{<}\right|}{|x|} e^{\mathrm{i} \theta}\right)\right\} & =-\sum_{m=1}^{\infty} \frac{1}{m} \frac{\left|x_{<}\right|^{m}}{|x|^{m}} \cos (m \theta) \\
& =-\frac{1}{2} \sum_{m=1}^{\infty} \frac{1}{m}\left\{\chi_{m}\left(x^{-1} x_{<}\right)-\frac{\left|x_{<}\right|^{2}}{|x|^{2}} \chi_{m-2}\left(x^{-1} x_{<}\right)\right\},
\end{aligned}
$$

where we have used Lemma 4.3 to express $\left(\left|x_{<}\right|^{m} /|x|^{m}\right) \cos (m \theta)$ in terms of the character functions $\chi_{m}$.

We now wish to obtain an expansion for the polyharmonic function

$$
\left|x-x_{<}\right|^{2 n} \ln \left|x-x_{<}\right|^{2} .
$$

To simplify this procedure, we observe that

$$
\left|x-x_{<}\right|^{2 n} \ln \left|x-x_{<}\right|^{2}=\left|x-x_{<}\right|^{2 n} \ln |x|^{2}+|x|^{2 n}\left|I-x^{-1} x_{<}\right|^{2 n} \ln \left|I-x^{-1} x_{<}\right|^{2},
$$

where $I$ is the $2 \times 2$ identity in $\mathcal{H}_{0}^{1}$ or the element $[1,0]$ in $\mathcal{C}^{2}$. This splits the function into a term containing the logarithmic singularity and a term amenable to "Laurent" expansion. We shall handle the two parts of the right hand side of (4.7) separately.

The coefficient of $\ln |x|$ in the expansion.

We first consider the polynomial that multiplies the $\ln |x|$ term in (4.7). We will give an expression for this polynomial in terms of the inner functions and discuss some symmetry properties.

Lemma 4.5. For $x, x_{<} \in \mathcal{R}^{4}$,

$\left|x-x_{<}\right|^{2 n}=\sum_{m=0}^{n}|x|^{2 m} \sum_{\ell=0}^{n-m} b_{m, \ell}^{n}\left|x_{<}\right|^{2 \ell} \chi_{n-m-\ell}\left(x_{<}{ }^{*} x\right)=\sum_{m=0}^{n}|x|^{2 m} \sum_{\ell=0}^{n-m} \sum_{i, j=0}^{n-m-\ell} D_{j, i}^{m, \ell}\left(x_{<}\right) t_{i, j}^{n-m-\ell}(x)$

where the coefficients $b_{m, \ell}^{n}$ are given recursively by

$$
b_{m, \ell}^{n+1}=b_{m-1, \ell}^{n}+b_{m, \ell-1}^{n}-b_{m, \ell}^{n}-b_{m-1, \ell-1}^{n}
$$

along with the initial conditions

$$
\begin{aligned}
& b_{m, \ell}^{0}= \begin{cases}1 & m=\ell=0, \\
0 & \text { otherwise, }\end{cases} \\
& b_{m, \ell}^{n}=0 \quad \text { if } m+\ell>n, \text { or } m, \ell \notin[0, n],
\end{aligned}
$$

and the coefficients $D_{j, i}^{m, \ell}\left(x_{<}\right)$are given by

$$
D_{j, i}^{m, \ell}\left(x_{<}\right)=b_{m, \ell}^{n}\left|x_{<}\right|^{2 \ell} t_{j, i}^{n-m-\ell}\left(x_{<}^{*}\right) .
$$


Proof. Simple application of (4.1b), along with the product rule (2.10) for character functions $\chi_{m}$, gives this first equality by induction on $n$. The second equality then follows by substituting (2.15) for $\chi_{n-m-\ell}\left(x_{<}^{*} x\right)$.

Remark 4.6. Since $b_{m, \ell}^{n}$ is real and

$$
t_{j, i}^{n-m-\ell}\left(x_{<}^{*}\right)=(-1)^{i+j} \overline{t_{n-m-\ell-j, n-m-\ell-i}^{n-m-\ell}\left(x<^{*}\right)}
$$

by symmetry (3.3) we see that

$$
D_{j, i}^{m, \ell}\left(x_{<}\right)=(-1)^{i+j} \overline{D_{n-m-\ell-j, n-m-\ell-i}^{m, \ell}\left(x_{<}\right)}
$$

for all $0 \leq m \leq n, 0 \leq \ell \leq n-m, 0 \leq i, j \leq n-m-\ell$. Provided the weights $d_{k}$ are real, this symmetry is inherited by the coefficients of polynomials

$$
q(x)=\sum_{k=0}^{N} d_{k}\left|x-x_{k}\right|^{2 n}=\sum_{m=0}^{n}|x|^{2 m} \sum_{\ell=0}^{n-m} \sum_{i, j=0}^{n-m-\ell} \widetilde{D}_{j, i}^{m, \ell} t_{i, j}^{n-m-\ell}(x)
$$

occurring as the coefficient of the $\ln |x|^{2}$ in the truncated far field expansion of Theorem 4.10 to come.

One use of this property would be to recast the polynomial $q(x)$ as the weighted sum of approximately half as many $t_{i, j}^{n-m-\ell}(x)$ 's, thereby reducing the operation count for approximate evaluation.

\section{The non-logarithmic part in the expansion.}

We now consider the infinite or far field part of (4.7). We find an explicit form for the expansion and give bounds on the error in approximation by truncation for this series.

Lemma 4.7. For $n \in \mathcal{N}_{0}$ and $y \in \mathcal{R}^{4},|y|<1$,

$$
|I-y|^{2 n} \ln |I-y|^{2}=\sum_{\ell=0}^{n+1} \sum_{m=\max \{1,2 \ell\}}^{\infty} c_{m, \ell}^{n}|y|^{2 \ell} \chi_{m-2 \ell}(y)
$$

where the series (4.11) converges absolutely. The coefficients $c_{m, \ell}^{n}$ are given by the formulae

$$
c_{m, \ell}^{0}=\left\{\begin{array}{cl}
-1 / m, & \ell=0, m \geq 1, \\
1 / m, & \ell=1, m-2 \ell \geq 0, \\
0, & \text { otherwise }
\end{array}\right.
$$

and the recurrence

$$
c_{m, \ell}^{n+1}=c_{m, \ell}^{n}-c_{m-1, \ell}^{n}-c_{m-1, \ell-1}^{n}+c_{m-2, \ell-1}^{n} .
$$

Proof. The proof is by induction on $n$.

Induction Basis: The case $n=0$ of formulae (4.11) and (4.12a) is contained in Lemma 4.4.

Induction Step: Assume (4.11) has been established for $n=K$. Then using the cosine formula (4.1a) and the product formula (2.10) we find

$$
\begin{aligned}
& |I-y|^{2 K+2} \ln |I-y|^{2} \\
& \quad=\sum_{\ell=0}^{K+1} \sum_{m=\max \{1,2 \ell\}}^{\infty} c_{m, \ell}^{K}|y|^{2 \ell}\left\{\chi_{m-2 \ell}(y)-\chi_{m+1-2 \ell}(y)-|y|^{2} \chi_{m-1-2 \ell}(y)+|y|^{2} \chi_{m-2 \ell}(y)\right\} .
\end{aligned}
$$


Rearranging by collecting terms of the same homogeneity we find a series

$$
|I-y|^{2 K+2} \ln |I-y|^{2}=\sum_{\ell=0}^{K+2} \sum_{m=\max \{1,2 \ell\}}^{\infty} c_{m, \ell}^{K+1}|y|^{2 \ell} \chi_{m-2 \ell}(y)
$$

converging absolutely for $|y|<1$ and with coefficients given by (4.12b). Thus (4.11) holds for $n=K+1$. The result follows by induction.

Lemma 4.8. For $m>2 n \geq 0$ and $0 \leq \ell \leq n+1$, the coefficients $c_{m, \ell}^{n}$ defined recursively in Lemma 4.7 have the explicit form

$$
c_{m, \ell}^{n}=(-1)^{n+\ell+1} n !\left(\begin{array}{c}
n+1 \\
\ell
\end{array}\right) / \prod_{\substack{k=m-\ell-n \\
k \neq m-2 \ell+1}}^{m-\ell+1} k
$$

Proof. The proof is by induction on $n$.

Induction Basis: The formula for $n=0$ is formula (4.12a) of Lemma 4.7.

Induction Step: Assume that the formula holds for $n=K$ and $m>2 K$. Then for $n=K+1$

$$
c_{m, \ell}^{K+1}=c_{m, \ell}^{K}-c_{m-1, \ell}^{K}-c_{m-1, \ell-1}^{K}+c_{m-2, \ell-1}^{K} .
$$

Assume now that $m>2(K+1)$ and $1 \leq \ell \leq K+1$. Using the induction hypothesis,

$$
\begin{aligned}
& c_{m, \ell}^{K+1}=(-1)^{K+1+\ell} K !\left\{\left(\begin{array}{c}
K+1 \\
\ell
\end{array}\right)\{(m-\ell-(K+1))(m-2 \ell+1)\right. \\
& -(m-\ell+1)(m-2 \ell)\}+\left(\begin{array}{c}
K+1 \\
\ell-1
\end{array}\right)\{(m-\ell-K-1)(m-2 \ell+2) \\
& -(m-\ell+1)(m-2 \ell+1)\}\} / \prod_{k=m-\ell-(K+1)}^{m-\ell+1} k \\
& =(-1)^{K+1+\ell} \frac{K !(K+1) !}{\ell !(K+2-\ell) !}\{(K+2-\ell)\{(m-\ell-K-1)(m-2 \ell+1) \\
& -(m-\ell-1)(m-2 \ell)\}+\ell\{(m-\ell-K-1)(m-2 \ell+2) \\
& -(m-\ell+1)(m-2 \ell+1)\}\} / \prod_{k=m-\ell-(K+1)}^{m-\ell+1} k \\
& =(-1)^{K+2+\ell} \frac{K !(K+1) !}{\ell !(K+2-\ell) !}(K+2)(K+1)(m-2 \ell+1) / \prod_{k=m-\ell-(K+1)}^{m-\ell+1} k
\end{aligned}
$$

agreeing with (4.13). The proof of the induction step when $\ell=0$ or $\ell=K+2$ is similar. Hence the result follows by induction.

Lemma 4.9. Let $n \in \mathcal{N}_{0}$ and $y \in \mathcal{R}^{4},|y|<1$. For $p \in \mathcal{N}$ let

$$
\widehat{g}_{p}(y)=\sum_{m=1}^{p} \sum_{\ell=0}^{\min \{\lfloor m / 2\rfloor, n+1\}} c_{m, \ell}^{n}|y|^{2 \ell} \chi_{m-2 \ell}(y)
$$

If $p>2 n$ then

$$
|| I-\left.y\right|^{2 n} \ln |I-y|^{2}-\widehat{g}_{p}(y) \mid \leq \frac{2^{n+1} n !(p+2)^{2}}{(p+1-n) \cdots(p-2 n)} \frac{|y|^{p+1}}{1-|y|}
$$


Proof. By Lemma 4.7,

$$
|| I-\left.y\right|^{2 n} \ln |I-y|^{2}-\widehat{g}_{p}(y)|\leq| \sum_{m=p+1}^{\infty} \sum_{\ell=0}^{n+1} c_{m, \ell}^{n}|y|^{2 \ell} \chi_{m-2 \ell}(y) \mid .
$$

Then using Lemma 4.8 the magnitude of all the terms of order $|y|^{m}$ can be estimated by

$$
\begin{aligned}
\left.\left|\sum_{\ell=0}^{n+1} c_{m, \ell}^{n}\right| y\right|^{2 \ell} \chi_{m-2 \ell}(y) \mid & \leq \sum_{\ell=0}^{n+1} \frac{(m-\ell-n-1) !}{(m-\ell+1) !}(m-2 \ell+1) n !\left(\begin{array}{c}
n+1 \\
\ell
\end{array}\right)(m-2 \ell+1)|y|^{m} \\
& \leq n !|y|^{m} \sum_{\ell=0}^{n+1} \frac{(m-2 \ell+1)^{2}}{(m-\ell+1) \cdots(m-\ell-n)}\left(\begin{array}{c}
n+1 \\
\ell
\end{array}\right), \\
& \leq n !|y|^{m} q_{n}(m) \sum_{\ell=0}^{n+1}\left(\begin{array}{c}
n+1 \\
\ell
\end{array}\right) \\
& =n ! 2^{n+1}|y|^{m} q_{n}(m),
\end{aligned}
$$

where

$$
q_{n}(m)=\frac{(m+1)^{2}}{(m-n)(m-(n+1)) \cdots(m-(2 n+1))}
$$

The function $q_{n}(m)$ is positive and decreasing in $m$ for $m>2 n+1$. Hence the right hand side of (4.15) can be estimated as

$$
\begin{aligned}
\left.\left|\sum_{m=p+1}^{\infty} \sum_{\ell=0}^{n+1} c_{m, \ell}^{n}\right| y\right|^{2 \ell} \chi_{m-2 \ell}(y) \mid & \leq\left.\sum_{m=p+1}^{\infty}\left|\sum_{\ell=0}^{n+1} c_{m, \ell}^{n}\right| y\right|^{2 \ell} \chi_{m-2 \ell}(y) \mid \\
& \leq \sum_{m=p+1}^{\infty} n ! 2^{n+1}|y|^{m} q_{n}(m) \\
& \leq 2^{n+1} n ! q_{n}(p+1) \frac{|y|^{p+1}}{1-|y|}
\end{aligned}
$$

\section{The full expansion and error bound.}

Combining the results in (4.7), Lemma 4.5 and Lemma 4.7, the function

$$
\Phi_{x_{<}}(x)=\left|x-x_{<}\right|^{2 n} \ln \left|x-x_{<}\right|^{2}
$$

may be approximated by the truncated series

$$
g_{p}(x)=\ln |x|^{2} \sum_{m=0}^{n}|x|^{2 m} \sum_{\ell=0}^{n-m} b_{m, \ell}^{n}\left|x_{<}\right|^{2 \ell} \chi_{n-m-\ell}\left(x^{*} x_{<}\right)+|x|^{2 n} \widehat{g}_{p}\left(x^{-1} x_{<}\right),
$$

where $\widehat{g}_{p}$ is defined in (4.14). Then from Lemma 4.9 we obtain the error bound

$$
\left|\Phi_{x_{<}}(x)-g_{p}(x)\right| \leq|x|^{2 n} \frac{2^{n+1} n !(p+2)^{2}}{(p+1-n) \cdots(p-2 n)} \frac{\left|x^{-1} x_{<}\right|^{p+1}}{1-\left|x^{-1} x_{<}\right|}
$$

for $|x|>\left|x_{<}\right|$. Since $p>2 n$ this bound is increasing in $|y|=\left|x_{<}\right| /|x|$. We can apply the bound to each centre $x_{k}$ in turn and sum. This gives 
Theorem 4.10. Suppose $x_{k} \in \mathcal{R}^{4},\left|x_{k}\right| \leq r$ and $d_{k} \in \mathcal{R}$ for each $1 \leq k \leq N$. Let $s$ be the $(n+2)$-harmonic spline

$$
s(x)=\sum_{k=1}^{N} d_{k}\left|x-x_{k}\right|^{2 n} \ln \left|x-x_{k}\right|^{2} .
$$

Furthermore, let $B_{m, \ell}$ be the $(n-m-\ell+1) \times(n-m-\ell+1)$ matrix

$$
\left[B_{i, j}^{m, \ell}\right]=b_{m, \ell}^{n} \sum_{k=1}^{N} d_{k}\left|x_{k}\right|^{2 \ell}\left(T_{n-m-\ell}\left(x_{k}^{*}\right)\right),
$$

and $C_{m, \ell}$ be the $(m-2 \ell+1) \times(m-2 \ell+1)$ matrix

$$
\left[C_{i, j}^{m, \ell}\right]=c_{m, \ell}^{n} \sum_{k=1}^{N} d_{k}\left|x_{k}\right|^{2 \ell}\left(T_{m-2 \ell}\left(x_{k}\right)\right),
$$

where the coefficients $b_{m, \ell}^{n}$ and $c_{m, \ell}^{n}$ are given recursively by (4.8) and (4.12) respectively. Let $p \in \mathcal{N}, p>2 n$, and set

$$
\begin{array}{r}
g_{p}(x)=\ln |x|^{2} \sum_{m=0}^{n} \sum_{\ell=0}^{n-m} \sum_{i, j=0}^{n-m-\ell} B_{j, i}^{m, \ell}|x|^{2 m} t_{i, j}^{n-m-\ell}(x) \\
\quad+\sum_{\ell=0}^{n+1} \sum_{m=\max \{1,2 \ell\}}^{p} \sum_{i, j=0}^{m-2 \ell} C_{j, i}^{m, \ell}|x|^{2(n+1-\ell)} o_{i, j}^{m-2 \ell}(x) \\
=\ln |x|^{2} \sum_{m=0}^{n}|x|^{2 m} \sum_{\ell=0}^{n-m} \operatorname{Tr}\left(B_{m, \ell} T_{n-m-\ell}(x)\right) \\
+\sum_{\ell=0}^{n+1} \sum_{m=\max \{1,2 \ell\}}^{p}|x|^{2(n+1-\ell)} \operatorname{Tr}\left(C_{m, \ell} O_{m-2 \ell}(x)\right)
\end{array}
$$

$x \in \mathcal{R}^{4} \backslash\{0\}$. Then for all $x$ with $|x|>r$

$$
\left|s(x)-g_{p}(x)\right| \leq M r^{2 n} \frac{(p+2)^{2} 2^{n+1} n !}{(p+1-n) \cdots(p-2 n)}\left(\frac{1}{c}\right)^{p-2 n+1} \frac{1}{1-1 / c},
$$

where $M=\sum_{k=1}^{N}\left|d_{k}\right|$ and $c=|x| / r$.

\section{Uniqueness.}

In this section we will prove that the truncated expansions, $g_{p}$, appearing in (4.4) and (4.17) are the only functions of these forms achieving the stated asymptotic accuracy in approximating $s$ as $|x| \rightarrow \infty$. These uniqueness results will allow us to form far field expansions in an inexpensive indirect manner, knowing that the expansions so obtained are identical with, and enjoy the same error estimates as, the computationally expensive directly formed expansions.

Lemma 5.1. Let $p \in \mathcal{N}_{0}$. Suppose a function $\widetilde{g}_{p}$ defined for $x \in \mathcal{R}^{4} \backslash\{0\}$ can be written in the form

$$
\begin{aligned}
\tilde{g}_{p}(x)=\ln |x|^{2} \sum_{m=0}^{n} \sum_{\ell=0}^{n-m} \sum_{i, j=0}^{n-m-\ell} \widetilde{B}_{j, i}^{m, \ell}|x|^{2 m} t_{i, j}^{n-m-\ell}(x) \\
+\sum_{\ell=0}^{n+1} \sum_{m=\max \{1,2 \ell\}}^{p} \sum_{i, j=0}^{m-2 \ell} \widetilde{C}_{j, i}^{m, \ell}|x|^{2(n+1-\ell)} o_{i, j}^{m-2 \ell}(x),
\end{aligned}
$$

where the various coefficients are complex numbers. Then 
(i) The coefficients $\left\{\widetilde{B}_{j, i}^{m, \ell}\right\}$ and $\left\{\widetilde{C}_{j, i}^{m, \ell}\right\}$ are uniquely determined by the function $\widetilde{g}_{p}$.

(ii) If $p>2 n$ and

then $\widetilde{g}_{p}$ is identically zero.

$$
\left|\widetilde{g}_{p}(x)\right|=o\left(|x|^{2 n-p}\right), \quad \text { as }|x| \rightarrow \infty,
$$

Proof. We will need to use the fundamental properties of the inner and outer functions developed in Section 3. Recall that $t_{i, j}^{m}$ is homogeneous of order $m$ and $\left\{t_{i, j}^{m}: 0 \leq i, j \leq m, 0 \leq m \leq q\right\}$ is an orthogonal set of non-trivial spherical harmonics on the unit sphere $\mathcal{S}^{3}$. The definition of the outer functions (2.12)

$$
o_{i, j}^{m}(z, w)=|x|^{-(2 m+2)} t_{i, j}^{m}(\bar{z},-w)
$$

then implies that $o_{i, j}^{m}$ is homogeneous of order $-(m+2)$ and $\left\{o_{i, j}^{m}: 0 \leq i, j \leq m, 0 \leq m \leq q\right\}$ is linearly independent on $\mathcal{S}^{3}$.

Now fix $p \in \mathcal{N}_{0}$ and consider a function of the form (5.1). Rearrange the finite sum $\tilde{g}_{p}$ by grouping together terms of the same growth at infinity, and arranging the groups in order of decreasing growth at infinity. The order of magnitude of $\widetilde{g}_{p}$ as $|x| \rightarrow \infty$ will be the same as that of the first non-zero group of terms.

Fix an integer $k$ and denote the sum of the group of terms of growth $|x|^{k} \ln |x|$ by $L_{k}$. Thus

$$
L_{k}(x)=\ln |x|^{2} \sum_{m=0}^{n} \sum_{\ell=0}^{n-m} \sum_{i, j=0}^{n-m-\ell} \delta_{k, n+m-\ell} \widetilde{B}_{j, i}^{m, \ell}|x|^{2 m} t_{i, j}^{n-m-\ell}(x) .
$$

Restricting attention to those terms for which the delta function is non-zero we see that among these a particular inner function $t_{i^{\prime}, j^{\prime}}^{k^{\prime}}$ can arise only when $k^{\prime}=k-2 m$ and thus can arise at most once. Hence by the linear independence of $\left\{t_{i, j}^{m}: 0 \leq i, j \leq m, 0 \leq m \leq n\right\}$ on $\mathcal{S}^{3}, L_{k}(x)$ is identically zero for all $x \neq 0$ if and only if

$$
\delta_{k, n+m-\ell} \widetilde{B}_{j, i}^{m, \ell}=0,
$$

for all $m=0, \ldots, n ; \ell=0, \ldots, n-m ; i, j=0, \ldots, n-m-\ell$. Similarly, the sum of the group of terms of growth $|x|^{k}$ at infinity is

$$
G_{k}(x)=\sum_{\ell=0}^{n+1} \sum_{m=\max \{1,2 \ell\}}^{p} \sum_{i, j=0}^{m-2 \ell} \delta_{k, 2 n-m} \widetilde{C}_{j, i}^{m, \ell}|x|^{2(n+1-\ell)} o_{i, j}^{m-2 \ell} .
$$

Fix $k$ and restrict attention to those terms for which the delta function is non zero. A particular outer function $o_{i^{\prime}, j^{\prime}}^{m^{\prime}}$ can arise only when $m^{\prime}=2 n-k-2 \ell$. Since $k$ and $n$ are fixed this happens for at most one value of $\ell$, and hence at most once. Thus by the linear independence of $\left\{o_{i^{\prime}, j^{\prime}}^{m^{\prime}}\right.$ : $\left.0 \leq i^{\prime}, j^{\prime} \leq m^{\prime}, 0 \leq m^{\prime} \leq q\right\}$ on $\mathcal{S}^{3}, G_{k}(x)$ is identically zero for all $x \neq 0$ if and only if

$$
\delta_{k, 2 n-m} \widetilde{C}_{j, i}^{m, \ell}=0
$$

for all $\ell=0, \ldots, n+1 ; m=\max \{1,2 \ell\}, \ldots, p ; i, j=0, \ldots, m$.

For (ii) just note that if $p>2 n$ then $o\left(|x|^{2 n-p}\right) \rightarrow 0$ so no terms can appear in the $\ln |x|^{2}$ summand, as each of those terms do not decay. Each term in the second summand is homogeneous of order $2 n-m \geq 2 n-p$. But the decay rate $o\left(|x|^{2 n-p}\right)$ precludes these terms from occurring, i.e., $\tilde{g}_{p}=0$.

A simpler argument based on the same ideas shows

Lemma 5.2. Let $p \in \mathcal{N}_{0}$. Suppose a function $\widetilde{g}_{p}$ defined for $x \in \mathcal{R}^{4} \backslash\{0\}$ can be written in the form

$$
\tilde{g}_{p}(x)=\sum_{m=0}^{p} \sum_{i, j=0}^{m} \widetilde{C}_{j, i}^{m} o_{i, j}^{m}(x)
$$

Then 
(i) The coefficients $\left\{\widetilde{C}_{j, i}^{m}\right\}$ are uniquely determined by the function $\widetilde{g}_{p}$.

(ii) If

$$
\left|\tilde{g}_{p}(x)\right|=o\left(|x|^{-(p+2)}\right), \quad \text { as }|x| \rightarrow \infty
$$

then $\widetilde{g}_{p}$ is identically zero.

\section{Translation of expansions.}

In this section we develop formulae which enable us to obtain a truncated expansion about one centre indirectly from a truncated expansion about another. The operation count for this translation operation depends only on the order of the original expansion, not upon the number of centres $x_{k}$ underlying it. In contrast, the operation count for direct expansion of a cluster, is $\mathcal{O}\left(N(n+1) p^{3}\right)$ where $N$ is the number of centres in the cluster. Thus indirect formation of expansions can be more efficient than direct expansion when the number of centres in a particular cluster is large and truncated expansions of sub-clusters are available.

For any matrix $A=\left(a_{i^{\prime}, j^{\prime}}\right)$, denote by $\left.A\right|_{i, j} ^{m}$ the $(m+1) \times(m+1)$ sub-matrix of $A$ which begins at the $(i, j)$ position, i.e.,

$$
\left(A_{i, j}^{m}\right)_{i^{\prime}, j^{\prime}}=a_{i+i^{\prime}, j+j^{\prime}}, \quad i^{\prime}, j^{\prime}=0, \ldots, m .
$$

Theorem 6.1 (Outer to outer or inner translation). Let $x, x_{<} \in \mathcal{H}_{0}^{1}$ be such that $0<\left|x_{<}\right|<$ $|x|$. Then

$$
o_{i, j}^{m L}\left(x-x_{<}\right)=\sum_{m^{\prime}=0}^{\infty} \operatorname{Tr}\left(\left.E\left(m, m^{\prime}, i\right) O_{m+m^{\prime}}(x)\right|_{i, j} ^{m^{\prime}} T_{m^{\prime}}\left(x_{<}\right)\right),
$$

where $E\left(m, m^{\prime}, i\right)$ is the $\left(m^{\prime}+1\right) \times\left(m^{\prime}+1\right)$ diagonal matrix with entries

$$
e\left(m, m^{\prime}, i\right)_{i^{\prime}, i^{\prime}}=\left(\begin{array}{c}
i+i^{\prime} \\
i
\end{array}\right)\left(\begin{array}{c}
m+m^{\prime}-\left(i+i^{\prime}\right) \\
m-i
\end{array}\right) .
$$

Proof. Using Lemma 4.1, Lemma 2.9 and the relationship (3.17) between the outer functions and the operators $R_{i, j}^{m}$,

$$
\begin{aligned}
o_{i, j}^{m}\left(x-x_{<}\right)=R_{i, j}^{m} \frac{1}{\left|x-x_{<}\right|^{2}} & =R_{i, j}^{m} \sum_{m^{\prime}=0}^{\infty} \operatorname{Tr}\left(O_{m^{\prime}}(x) T_{m^{\prime}}\left(x_{<}\right)\right) \\
& =R_{i, j}^{m} \sum_{m^{\prime}=0}^{\infty} \sum_{i^{\prime}, j^{\prime}=0}^{m^{\prime}} o_{i^{\prime}, j^{\prime}}^{m^{\prime}}(x) t_{j^{\prime}, i^{\prime}}^{m^{\prime}}\left(x_{<}\right) \\
& =R_{i, j}^{m} \sum_{m^{\prime}=0}^{\infty} \sum_{i^{\prime}, j^{\prime}=0}^{m^{\prime}} t_{j^{\prime}, i^{\prime}}^{m^{\prime}}\left(x_{<}\right) R_{i^{\prime}, j^{\prime}}^{m^{\prime}} \frac{1}{|x|^{2}} \\
& =\sum_{m^{\prime}=0}^{\infty} \sum_{i^{\prime}, j^{\prime}=0}^{m^{\prime}} t_{j^{\prime}, i^{\prime}}^{m^{\prime}}\left(x_{<}\right) R_{i, j}^{m} R_{i^{\prime}, j^{\prime}}^{m^{\prime}} \frac{1}{|x|^{2}} \\
& =\sum_{m^{\prime}=0}^{\infty} \sum_{i^{\prime}, j^{\prime}=0}^{m^{\prime}} t_{j^{\prime}, i^{\prime}}^{m^{\prime}}\left(x_{<}\right) e\left(m, m^{\prime}, i\right)_{i^{\prime}, i^{\prime}} R_{i+i^{\prime}, j+j^{\prime}}^{m+m^{\prime}} \frac{1}{|x|^{2}} \\
& =\sum_{m^{\prime}=0}^{\infty} \sum_{i^{\prime}, j^{\prime}=0}^{m^{\prime}} e\left(m, m^{\prime}, i\right)_{i^{\prime}, i^{\prime}} o_{i+i^{\prime}, j+j^{\prime}}^{m+m^{\prime}}(x) t_{j^{\prime}, i^{\prime}}^{m^{\prime}}\left(x_{<}\right) \\
& =\sum_{m^{\prime}=0}^{\infty} \operatorname{Tr}\left(\left.E\left(m, m^{\prime}, i\right) O_{m+m^{\prime}}(x)\right|_{i, j} ^{m^{\prime}} T_{m^{\prime}}\left(x_{<}\right)\right)
\end{aligned}
$$

where the differentiation term by term is justified by the real analyticity. 
This Theorem is sufficient to translate a far field expansion of the type in (4.4). In particular consider an expansion like (4.4) but centred on $x_{<} \neq 0$. Then

$$
\begin{aligned}
g_{p}\left(x-x_{<}\right) & =\sum_{m=0}^{p} \sum_{i, j=0}^{m} C_{j, i}^{m} o_{i, j}^{m}\left(x-x_{<}\right) \\
& =\sum_{m=0}^{p} \sum_{i, j=0}^{m} C_{j, i}^{m} \sum_{m^{\prime}=0}^{\infty} \sum_{i^{\prime}, j^{\prime}=0}^{m^{\prime}} e\left(m, m^{\prime}, i\right)_{i^{\prime}, i^{\prime}} o_{i+i^{\prime}, j+j^{\prime}}^{m+m^{\prime}}(x) t_{j^{\prime}, i^{\prime}}^{m^{\prime}}\left(x_{<}\right) \\
& =\sum_{m=0}^{p} \sum_{i, j=0}^{m} D_{j, i}^{m} o_{i, j}^{m}(x)+\mathcal{O}\left(|x|^{-(p+\overline{3})}\right)
\end{aligned}
$$

where the coefficients $D_{j, i}^{m}$ are defined by the "convolution"

$$
\left(\begin{array}{c}
m \\
i
\end{array}\right) D_{j, i}^{m}=\sum_{m^{\prime}=0}^{m}\left(\begin{array}{c}
m \\
m^{\prime}
\end{array}\right) \sum_{i^{\prime}, j^{\prime}=0}^{m^{\prime}}\left(\begin{array}{c}
m-m^{\prime} \\
i-i^{\prime}
\end{array}\right) t_{j-j^{\prime}, i-i^{\prime}}^{m-m^{\prime}}\left(x_{<}\right)\left(\begin{array}{c}
m^{\prime} \\
i^{\prime}
\end{array}\right) C_{j^{\prime}, i^{\prime}}^{m^{\prime}}
$$

Thus

$$
h_{p}(x)=\sum_{m=0}^{p} \sum_{i, j=0}^{m} D_{j, i}^{m} o_{i, j}^{m}(x)
$$

approximates $g_{p}(x)$ with error of order $\mathcal{O}\left(|x|^{-(p+3)}\right)$ as $|x| \rightarrow \infty$. But by Theorem 4.2 the series formed directly, $u_{p}$, is of the same form and shares the same order of approximation. Hence the difference $u_{p}(x)-h_{p}(x)$ is $\mathcal{O}\left(|x|^{-(p+3)}\right)$ as $|x| \rightarrow \infty$, and by the Uniqueness Theorem, Lemma 5.2, $u_{p}$ and $h_{p}$ are identical.

Furthermore, Theorem 6.1 is sufficient to translate an expansion of the form (6.2) into a Taylor series about 0 (a Maclaurin series). Since the functions $o_{i, j}^{m}$ are homogenous of degree $m$,

$$
o_{i, j}^{m}\left(x-x_{<}\right)=(-1)^{m} o_{i, j}^{m}\left(x_{<}-x\right) .
$$

This simple observation allows the roles of $x$ and $x_{<}$to be switched in the application of Theorem 6.1. Starting with $g_{p}$ defined by (6.2) and proceeding in this manner, we obtain

$$
\begin{aligned}
g_{p}\left(x-x_{<}\right) & =\sum_{m^{\prime}=0}^{p} \sum_{i^{\prime}, j^{\prime}=0}^{m^{\prime}} C_{j^{\prime}, i^{\prime}}^{m^{\prime}}(-1)^{m^{\prime}} o_{i^{\prime}, j^{\prime}}^{m^{\prime}}\left(x_{<}-x\right) \\
& =\sum_{m^{\prime}=0}^{p} \sum_{i^{\prime}, j^{\prime}=0}^{m^{\prime}}(-1)^{m^{\prime}} C_{j^{\prime}, i^{\prime}}^{m^{\prime}} \sum_{m=0}^{\infty} \sum_{i, j=0}^{m} e\left(m^{\prime}, m, i^{\prime}\right)_{i, i} o_{i+i^{\prime}, j+j^{\prime}}^{m+m^{\prime}}\left(x_{<}\right) t_{j, i}^{m}(x) \\
& =\sum_{m=0}^{p} \sum_{i, j=0}^{m} F_{j, i}^{m} t_{i, j}^{m}(x)+\mathcal{O}\left(|x|^{p+1}\right), \quad \text { as }|x| \rightarrow 0,
\end{aligned}
$$

where the coefficients $F_{j, i}^{m}$ are given by the "correlation"

$$
\left(\begin{array}{c}
m \\
j
\end{array}\right)^{-1} F_{j, i}^{m}=\sum_{m^{\prime}=0}^{p}\left(\begin{array}{c}
m+m^{\prime} \\
m
\end{array}\right) \sum_{i^{\prime}, j^{\prime}=0}^{m^{\prime}}\left(\begin{array}{c}
m+m^{\prime} \\
j+j^{\prime}
\end{array}\right)^{-1} o_{j+j^{\prime}, i+i^{\prime}}^{m+m^{\prime}}\left(x_{<}\right)(-1)^{m^{\prime}}\left(\begin{array}{c}
m^{\prime} \\
j^{\prime}
\end{array}\right) C_{i^{\prime}, j^{\prime}}^{m^{\prime}}
$$

Then by the characterisation of the Maclaurin polynomial $q$ of degree $p$ for a function $f$ as the only polynomial of total degree $p$ with

$$
|f(x)-q(x)|=o\left(|x|^{p}\right), \quad \text { as }|x| \rightarrow 0,
$$

it follows that

$$
q(\cdot)=\sum_{m=0}^{p} \sum_{i, j=0}^{m} F_{j, i}^{m} t_{i, j}^{m}(\cdot)
$$


Theorem 6.2 (Inner-to-inner translation formula). For all $x, x_{<} \in \mathcal{R}^{4}, m \in \mathcal{N}_{0}$ and $0 \leq$ $i, j \leq m$,

$$
t_{i, j}^{m}\left(x-x_{<}\right)=\sum_{m^{\prime}=0}^{m} \sum_{\substack{i^{\prime}=\max \left\{0, i-\left(m-m^{\prime}\right)\right\} \\
j^{\prime}=\max \left\{0, j-\left(m-m^{\prime}\right)\right\}}}^{\substack{i^{\prime}=\min \left\{i, m^{\prime}\right\} \\
j^{\prime}=\min \left\{j, m^{\prime}\right\}}}(-1)^{m-m^{\prime}}\left(\begin{array}{c}
m-j \\
m^{\prime}-j^{\prime}
\end{array}\right)\left(\begin{array}{c}
j \\
j^{\prime}
\end{array}\right) t_{i-i^{\prime}, j-j^{\prime}}^{m-m^{\prime}}\left(x_{<}\right) t_{i^{\prime}, j^{\prime}}^{m i^{\prime}}(x) .
$$

Proof. Because the functions $\left\{t_{i^{\prime}, j^{\prime}}^{m^{\prime}}: 0 \leq i^{\prime}, j^{\prime} \leq m^{\prime}, 0 \leq m^{\prime} \leq m\right\}$ form a basis for harmonic homogenous polynomials of degree at most $m$, and since $t_{i, j}^{m}\left(\cdot-x_{<}\right)$is such a polynomial,

$$
t_{i, j}^{m}\left(x-x_{<}\right)=\sum_{m^{\prime}=0}^{m} \sum_{i^{\prime}, j^{\prime}=0}^{m^{\prime}} a_{i, i^{\prime}, j, j^{\prime}}^{m, m^{\prime}} t_{i^{\prime}, j^{\prime}}^{m^{\prime}}(x)
$$

for some coefficients $a_{i, i^{\prime}, j, j^{\prime}}^{m n, m^{\prime}}$ that depend on $x_{<}$. Applying the functionals $\lambda_{i^{\prime \prime}, j^{\prime \prime}}^{m^{\prime \prime}}$ of Lemma 3.10 to this expression gives

$$
\left(\begin{array}{c}
j \\
j^{\prime \prime}
\end{array}\right)\left(\begin{array}{c}
m-j \\
m^{\prime \prime}-j^{\prime \prime}
\end{array}\right) t_{i-i^{\prime \prime}, j-j^{\prime \prime}}^{m-m^{\prime \prime}}\left(-x_{<}\right)=a_{i, i^{\prime \prime}, j, j^{\prime \prime}}^{m, m^{\prime \prime}}
$$

Since $(-1)^{m-m^{\prime \prime}}$ factors out by the homogeneity of $t_{i-i^{\prime \prime}, j-j^{\prime \prime}}^{m-m^{\prime \prime}}$, the result follows once we recall that the left hand side is zero unless $j^{\prime \prime} \leq j, j-j^{\prime \prime} \leq m-m^{\prime \prime}, i^{\prime \prime} \leq i$ and $i-i^{\prime \prime} \leq m-m^{\prime \prime}$.

This theorem may be used to translate a polynomial expansion such as (6.5). For example, if

$$
q(x)=\sum_{m^{\prime}=0}^{p} \sum_{i^{\prime}, j^{\prime}=0}^{m^{\prime}} F_{j^{\prime}, i^{\prime}}^{m^{\prime}} t_{i^{\prime}, j^{\prime}}^{m^{\prime}}\left(x-x_{<}\right),
$$

then by Theorem 6.2 , we get

$$
\begin{aligned}
q(x) & =\sum_{m^{\prime}=0}^{p} \sum_{i^{\prime}, j^{\prime}=0}^{m^{\prime}} F_{j^{\prime}, i^{\prime}}^{m^{\prime}} \sum_{m=0}^{m^{\prime}} \sum_{i, j=0}^{m}(-1)^{m^{\prime}-m}\left(\begin{array}{c}
m^{\prime}-j^{\prime} \\
m-j
\end{array}\right)\left(\begin{array}{c}
j^{\prime} \\
j
\end{array}\right) t_{i^{\prime}-i, j^{\prime}-j}^{m^{\prime}-m}\left(x_{<}\right) t_{i, j}^{m}(x) \\
& =\sum_{m=0}^{p} \sum_{i, j=0}^{m} G_{j, i}^{m} t_{i, j}^{m}(x),
\end{aligned}
$$

where the coefficients $G_{j, i}^{m}$ are given by the "convolution"

$$
\left(\begin{array}{c}
m \\
j
\end{array}\right)^{-1} G_{j, i}^{m}=\sum_{m^{\prime}=0}^{p}\left(\begin{array}{c}
m^{\prime} \\
m
\end{array}\right) \sum_{i^{\prime}, j^{\prime}=0}^{m^{\prime}}\left(\begin{array}{c}
m^{\prime} \\
j^{\prime}
\end{array}\right)^{-1} F_{j^{\prime}, i^{\prime}}^{m^{\prime}}(-1)^{m^{\prime}-m}\left(\begin{array}{c}
m^{\prime}-m \\
j^{\prime}-j
\end{array}\right) t_{i^{\prime}-i, j^{\prime}-j}^{m^{\prime}-m}\left(x_{<}\right) .
$$

It should be noted that this is an exact recentering of the polynomial $q$.

Just as we were able to translate expansions of the form (6.2), we want to be able to translate expansions like (4.17). One of our tools will be formulae for the products of $z, w, \bar{z}$ or $\bar{w}$ with a single inner or single outer function. These multiplication rules are contained in Lemmas 6.3 and 6.4 below.

Lemma 6.3. For $m \geq 0$ and $0 \leq i, j \leq m$,

$$
\begin{aligned}
z o_{i, j}^{m}(z, w) & =\frac{i+1}{m+1}|x|^{2} o_{i+1, j+1}^{m+1}(z, w)+\frac{m-j}{m+1} o_{i, j}^{m-1}(z, w) \\
w o_{i, j}^{m}(z, w) & =-\frac{m+1-i}{m+1}|x|^{2} o_{i, j+1}^{m+1}(z, w)+\frac{m-j}{m+1} o_{i-1, j}^{m-1}(z, w) \\
\bar{z} o_{i, j}^{m}(z, w) & =\frac{m+1-i}{m+1}|x|^{2} o_{i, j}^{m+1}(z, w)+\frac{j}{m+1} o_{i-1, j-1}^{m-1}(z, w) \\
\bar{w} o_{i, j}^{m}(z, w) & =\frac{i+1}{m+1}|x|^{2} o_{i+1, j}^{m+1}(z, w)-\frac{j}{m+1} o_{i, j-1}^{m-1}(z, w) .
\end{aligned}
$$


Proof. First assume $m>0$. Differentiate (2.13) with respect to $\bar{z}$. For the right hand side we obtain

$$
\begin{aligned}
(m-j) z_{1}\left(z_{1} \bar{z}+z_{2} \bar{w}\right)^{m-1-j}\left(z_{1}(-w)+z_{2} z\right)^{j} & =(m-j) z_{1}|x|^{2 m} \sum_{i=0}^{m-1} z_{1}^{m-1-i} z_{2}^{i} o_{i, j}^{m-1}(z, w) \\
& =(m-j)|x|^{2 m} \sum_{i=0}^{m-1} z_{1}^{m-i} z_{2}^{i} o_{i, j}^{m-1}(z, w) .
\end{aligned}
$$

Since $|x|^{2}=z \bar{z}+w \bar{w}$, for the left hand side we have

$$
\begin{aligned}
& z(m+1)|x|^{2 m} \sum_{i=0}^{m} z_{1}^{m-i} z_{2}^{i} o_{i, j}^{m}(z, w)+|x|^{2(m+1)} \sum_{i=0}^{m} z_{1}^{m-i} z_{2}^{i} \frac{\partial}{\partial \bar{z}} o_{i, j}^{m}(z, w) \\
& =z(m+1)|x|^{2 m} \sum_{i=0}^{m} z_{1}^{m-i} z_{2}^{i} o_{i, j}^{m}(z, w)-|x|^{2(m+1)} \sum_{i=0}^{m} z_{1}^{m-i} z_{2}^{i}(i+1) o_{i+1, j+1}^{m+1}(z, w)
\end{aligned}
$$

where we have used (3.13d) to evaluate $\frac{\partial}{\partial \bar{z}} o_{i, j}^{m}$. By considering the coefficient of $z_{1}^{m-i} z_{2}^{i}$ in (6.9) and (6.10) we see that

$$
(m-j) o_{i, j}^{m-1}(z, w)=z(m+1) o_{i, j}^{m}(z, w)-|x|^{2}(i+1) o_{i+1, j+1}^{m+1}(z, w)
$$

for $0 \leq i, j \leq m$, which proves $(6.8 \mathrm{a})$

By differentiating (2.13) with respect to $\bar{w}, z$ and $w$, in a similar manner we obtain (6.8b), (6.8c) and (6.8d) respectively, for $m>0$. The special case of $m=0$ for (6.8) follows directly from $o_{0,0}^{0}=|x|^{-2}$ and the recurrence relations (3.8).

Substituting (2.12) into (6.8) leads to a similar result for the inner functions. Specifically,

Lemma 6.4. For $m \geq 0$ and $0 \leq i, j \leq m$,

$$
\begin{aligned}
& z t_{i, j}^{m}(z, w)=\frac{m+1-i}{m+1} t_{i, j}^{m+1}(z, w)+\frac{j}{m+1}|x|^{2} t_{i-1, j-1}^{m-1}(z, w), \\
& w t_{i, j}^{m}(z, w)=\frac{m+1-i}{m+1} t_{i, j+1}^{m+1}(z, w)-\frac{m-j}{m+1}|x|^{2} t_{i-1, j}^{m-1}(z, w), \\
& \bar{z} t_{i, j}^{m}(z, w)=\frac{i+1}{m+1} t_{i+1, j+1}^{m+1}(z, w)+\frac{m-j}{m+1}|x|^{2} t_{i, j}^{m-1}(z, w), \\
& \bar{w} t_{i, j}^{m}(z, w)=-\frac{i+1}{m+1} t_{i+1, j}^{m+1}(z, w)+\frac{j}{m+1}|x|^{2} t_{i, j-1}^{m-1}(z, w) .
\end{aligned}
$$

Since $t_{i, j}^{m}=0$ and $o_{i, j}^{m}=0$ if $m<0$, multiple applications of Lemma 6.3 and Lemma 6.4 may be used to obtain

Corollary 6.5. Let $p$ be a given homogenous polynomial of degree $m^{\prime}$ in $z, \bar{z}, w$ and $\bar{w}$. Then to each inner function $t_{i, j}^{m}$ there correspond constants $\left\{F_{i^{\prime}, j^{\prime}}^{\ell^{\prime}}\right\}$ and to each outer function $o_{i, j}^{m}$ there correspond constants $\left\{G_{i^{\prime}, j^{\prime}}^{\ell^{\prime}}\right\}$ such that

$$
\begin{gathered}
p(z, \bar{z}, w, \bar{w}) t_{i, j}^{m}(x)=\sum_{\ell^{\prime}=0}^{\min \left\{m^{\prime},\left\lfloor\left(m+m^{\prime}\right) / 2\right\rfloor\right\}}|x|^{2 \ell^{\prime}} \sum_{i^{\prime}, j^{\prime}=0}^{m+m^{\prime}-2 \ell} F_{i^{\prime}, j^{\prime}}^{\ell^{\prime}} t_{i^{\prime}, j^{\prime}}^{m+m^{\prime}-2 \ell^{\prime}}(x), \\
p(z, \bar{z}, w, \bar{w}) o_{i, j}^{m}(x)=\sum_{\ell^{\prime}=0}^{\min \left\{m^{\prime},\left\lfloor\left(m+m^{\prime}\right) / 2\right\rfloor\right\}}|x|^{2\left(m^{\prime}-\ell^{\prime}\right)} \sum_{i^{\prime}, j^{\prime}=0}^{m+m^{\prime}-2 \ell^{\prime}} G_{i^{\prime}, j^{\prime}}^{\ell^{\prime}} o_{i^{\prime}, j^{\prime}}^{m+m^{\prime}-2 \ell^{\prime}}(x) .
\end{gathered}
$$


We now demonstrate how these results may be used to translate truncated a far field series, such as (4.17), due to a polyharmonic spline. Let $g_{p}$ be such a series with centre of expansion at $x_{<}$, i.e.,

$$
\begin{aligned}
g_{p}(x)=\ln \left|x-x_{<}\right|^{2} \sum_{\ell=0}^{n}\left|x-x_{<}\right|^{2 \ell}\left\{\sum_{m=0}^{n-\ell} \sum_{i, j=0}^{n-\ell-m} B_{j, i}^{\ell, m} t_{i, j}^{n-\ell-m}\left(x-x_{<}\right)\right\} \\
+\sum_{\ell=0}^{n+1}\left|x-x_{<}\right|^{2(n+1-\ell)}\left\{\sum_{m=\max \{1,2 \ell\}}^{p} \sum_{i, j=0}^{m-2 \ell} C_{j, i}^{\ell, m} o_{i, j}^{m-2 \ell}\left(x-x_{<}\right)\right\} .
\end{aligned}
$$

The translations of objects such as those in the two sets of curly braces has been discussed already. The terms in the first set may be translated via Theorem 6.2 in much the same way as (6.6) was translated. The terms in the second set of braces may be translated using Theorem 6.1 in a similar manner to (6.2). Let $\left\{\widetilde{B}_{j, i}^{\ell, m}\right\}$ and $\left\{\widetilde{C}_{j, i}^{\ell, m}\right\}$ be the translated coefficients. Then

$$
\begin{aligned}
g_{p}(x)= & \ln \left|x-x_{<}\right|^{2} \sum_{\ell=0}^{n}\left|x-x_{<}\right|^{2 \ell}\left\{\sum_{m=0}^{n-\ell} \sum_{i, j=0}^{n-\ell-m} \widetilde{B}_{j, i}^{\ell, m} t_{i, j}^{n-\ell-m}(x)\right\} \\
& +\sum_{\ell=0}^{n+1}\left|x-x_{<}\right|^{2(n+1-\ell)}\left\{\sum_{m=\max \{1,2 \ell\}}^{p} \sum_{i, j=0}^{m-2 \ell} \widetilde{C}_{j, i}^{\ell, m} o_{i, j}^{m-2 \ell}(x)\right\}+\mathcal{O}\left(|x|^{-(p+1-2 n)}\right) .
\end{aligned}
$$

Recall that

$$
\left|x-x_{<}\right|^{2}=|x|^{2}-2\left\langle x, x_{<}\right\rangle+\left|x_{<}\right|^{2}=|x|^{2}-\left(z \overline{z_{<}}+\bar{z} z_{<}+w \overline{w_{<}}+\bar{w} w_{<}\right)+\left|x_{<}\right|^{2} .
$$

Thus we may use Lemma 6.4 to "translate" any product of the form $\left|x-x_{<}\right|^{2} t_{i^{\prime}, j^{\prime}}^{m^{\prime}}(x)$ into a sum of at most ten terms of the form $|x|^{2 \ell^{\prime \prime}} t_{i^{\prime \prime}, j^{\prime \prime}}^{m^{\prime \prime}}(x)$, where the coefficients of those terms depend on $x_{<}$. An analogous procedure employing Lemma 6.3 translates a product of the form $\left|x-x_{<}\right|^{2} o_{i^{\prime}, j^{\prime}}^{m^{\prime}}(x)$ into a sum of at most ten terms of the form $|x|^{2 \ell^{\prime \prime}} o_{i^{\prime \prime}, j^{\prime \prime}}^{m^{\prime \prime}}(x)$. Applying this procedure repeatedly to $(6.13)$ viewed as a nested product

$$
f_{0}(x)+\left|x-x_{<}\right|^{2}\left(f_{1}(x)+\left|x-x_{<}\right|^{2}\left(f_{2}(x)+\cdots+\left|x-x_{<}\right|^{2}\left(f_{n}(x)+\left|x-x_{<}\right|^{2} f_{n+1}(x)\right) \cdots\right)\right)
$$

brings $g_{p}$ to the form

$$
\begin{aligned}
g_{p}(x)=\ln \left|x-x_{<}\right|^{2} \sum_{\ell=0}^{n}|x|^{2 \ell} \sum_{m=0}^{n-\ell} \sum_{i, j=0}^{n-\ell-m} \widetilde{\widetilde{B}}_{j, i}^{\ell, m} t_{i, j}^{n-\ell-m}(x) \\
+\sum_{\ell=0}^{n+1}|x|^{2(n+1-\ell)} \sum_{m=\max \{1,2 \ell\}} \sum_{i, j=0}^{m-2 \ell} \widetilde{\widetilde{C}}_{j, i}^{\ell, m} o_{i, j}^{m-2 \ell}(x)+\mathcal{O}\left(|x|^{-(p+1-2 n)}\right),
\end{aligned}
$$

with only the $\ln \left|x-x_{<}\right|^{2}$ term left untranslated. This particular step in the transiation costs $\mathcal{O}\left((n+1)^{2}(p+1)^{3}\right)$ operations. This is acceptable since $n$ is small, typically $n \leq 2$, and the number of terms in the series to be translated is $\mathcal{O}\left((n+1)(p+1)^{3}\right)$.

By Theorem 4.10,

$$
\ln \left|x-x_{<}\right|^{2}=\ln |x|^{2}+\sum_{\ell^{\prime}=0}^{1}|x|^{2-2 \ell^{\prime}} \sum_{m^{\prime}=\max \left\{1,2 \ell^{\prime}\right\}}^{p^{\prime}} \sum_{i^{\prime}, j^{\prime}=0}^{m^{\prime}-2 \ell^{\prime}} D_{j^{\prime}, i^{\prime}}^{\ell^{\prime}, m^{\prime}}\left(x_{<}\right) o_{i^{\prime}, j^{\prime}}^{m^{\prime}-2 \ell^{\prime}}(x)+\mathcal{O}\left(|x|^{-\left(p^{\prime}+1\right)}\right) .
$$


Substituting this into (6.14) gives

$$
\begin{aligned}
g_{p}(x)= & \ln |x|^{2} \sum_{\ell=0}^{n}|x|^{2 \ell} \sum_{m=0}^{n-\ell} \sum_{i, j=0}^{n-\ell-m} \widetilde{\widetilde{B}}_{j, i}^{\ell, m} t_{i, j}^{n-\ell-m}(x) \\
& +F_{x<}(x)+\sum_{\ell=0}^{n+1}|x|^{2(n+1-\ell)} \sum_{m=\max \{1,2 \ell\}}^{p} \sum_{i, j=0}^{m-2 \ell} \widetilde{\widetilde{C}}_{j, i}^{\ell, m} o_{i, j}^{m-2 \ell}(x)+\mathcal{O}\left(|x|^{-(p+1-2 n)}\right),
\end{aligned}
$$

where $F_{x_{<}}(x)$ is given by the product

$$
\left\{\sum_{\ell=0}^{n}|x|^{2 \ell} \sum_{m=0}^{n-\ell} \sum_{i, j=0}^{n-\ell-m} \widetilde{\widetilde{B}}_{j, i}^{\ell, m} t_{i, j}^{n-\ell-m}(x)\right\} \times\left\{\sum_{\ell^{\prime}=0}^{1}|x|^{2-2 \ell^{\prime}} \sum_{m^{\prime}=\max \left\{1,2 \ell^{\prime}\right\}}^{p^{\prime}} \sum_{i^{\prime}, j^{\prime}=0}^{m^{\prime}-2 \ell^{\prime}} D_{j^{\prime}, i^{\prime}}^{\ell^{\prime}, m^{\prime}}\left(x_{<}\right) o_{i^{\prime}, j^{\prime}}^{m^{\prime}-2 \ell^{\prime}}(x)\right\}
$$

after it has been truncated by removing terms that are $\mathcal{O}\left(|x|^{-(p+1-2 n)}\right)$ as $|x| \rightarrow \infty$. From Corollary $6.5,(6.16)$ can be written in the form

$$
\sum_{\ell=0}^{n+1}|x|^{2(n+1-\ell)} \sum_{m=\max \{1,2 \ell\}}^{p} \sum_{i, j=0}^{m-2 \ell} \widetilde{D}_{j, i}^{\ell, m} o_{i, j}^{m-2 \ell}(x) .
$$

This completes the translation.

\section{References}

[1] J. F. Adams, Lectures on Lie Groups, W. A. Benjamin, Inc., New York, 1969.

[2] S. L. Altmann, Rotations, Quaternions and Double Groups, Oxford University Press, 1986.

[3] R. K. Beatson and L. L. Greengard, A short course on fast multipole methods, in Wavelets, Multilevel Methods and Elliptic PDEs, M. Ainsworth, J. Levesley, W. Light, and M. Marletta, eds., Oxford Lniversity Press, 1997, pp. 1-37.

[4] R. K. Beatson And W. A. Light, Fast evaluation of radial basis functions: Methods for two-dimensional polyharmonic splines, IMA J. Numer. Anal., 17 (1997), pp. 343-372.

[5] R. K. Beatson and G. N. Newsam, Fast evaluation of radial basis functions: I, Computers Math. Applic., 24 (1992), pp. 7-19.

[6] - Fast evaluation of radial basis functions: Moment based methods, SIAM J. Scientific Comput., 19 (1998), pp. 1428-1449.

[7] R. K. Beatson, A. M. TAN, AND M. J. D. Powell, Fast evaluation of radial basis functions: Methods for 3-dimensional polyharmonic splines. In preparation.

[8] M. A. EPTON AND B. DEvBart, Multipole translation theory for the three-dimensional Laplace and Helmholtz equations, SIAM J. Sci. Comput., 16 (1995), pp. 865-897.

[9] J. Flusser, An adaptive method for image registration, Pattern Recognition, 25 (1992), pp. $45-54$.

[10] R. Franke, Scattered data interpolation: Tests of some methods, Math. Comp., 38 (1982), pp. 181-200.

[11] L. L. GREengaRd and V. Rokhlin, A fast algorithm for particle simulations, J. Comput. Phys, 73 (1987), pp. 325-348. 
[12] E. Hewitr AND K. A. Ross, Abstract Harmonic Analysis, II, vol. 152 of Grundlehren der mathematischen Wissenschaften, Springer-Verlag, Berlin, 1970.

[13] M. F. HUTCHINSON, The application of thin plate smoothing splines to continent-wide data assimilation, in Data Assimilation Systems, J. D. Jasper, ed., BRMC Research Report No. 27, Melbourne, 1991, Bureau of Meteorology, pp. 104-113.

[14] A. Kyrala., Theoretical Physics: Applications of Vectors, Matrices, Tensors and Quaternions, W. B. Saunders Company, Philadelphia, 1967.

[15] C. MülLER, Spherical Harmonics, vol. 17 of Lecture Notes in Mathematics, Springer-Verlag, New York, 1966.

[16] M. A. NAimark ANd A. I. STERn, Theory of Group Representations, vol. 246 of Grundlehren der mathematischen Wissenschaften, Springer-Verlag, New York, 1982.

[17] M. J. D. Powell, The theory of radial basis function approximation in 1990, in Advances in Numerical Analysis II: Wavelets, subdivision algorithms and radial functions, W. Light, ed., Oxford University Press, 1992, pp. 105-210.

[18] D. L. RAGozin, Constructive polynomial approximation on spheres and projective spaces, Trans. Amer. Math. Soc., 162 (1971), pp. 157-170.

[19] —- Uniform convergence of spherical harmonic expansions, Math. Ann., 195 (1972), pp. 87-94.

[20] R. Sibson and G. Stone, Computation of thin-plate splines, SIAM J. Scientific and Statistical Computing, 12 (1991), pp. 1304-1313.

[21] G. TURK AND J. F. O'BRIEN, Shape transformation using variational implicit surfaces, in SIGGRAPH'99, Aug 1999, pp. 335-342. 


\section{A A table of inner and outer functions.}

$$
\begin{aligned}
& T_{0}=[1] \\
& T_{1}=\left[\begin{array}{cc}
z & w \\
-\bar{w} & \bar{z}
\end{array}\right] \\
& T_{2}=\left[\begin{array}{ccc}
z^{2} & z w & w^{2} \\
-2 z \bar{w} & z \bar{z}-\bar{w} w & 2 w \bar{z} \\
\bar{w}^{2} & -\overline{w z} & \bar{z}^{2}
\end{array}\right] \\
& T_{3}=\left[\begin{array}{cccc}
z^{3} & z^{2} w & z w^{2} & w^{3} \\
-3 z^{2} \bar{w} & z^{2} \bar{z}-2 \bar{w} z w & 2 z w \bar{z}-\bar{w} w^{2} & 3 w^{2} \bar{z} \\
3 z \bar{w}^{2} & -2 z \overline{w z}+\bar{w}^{2} w & z \bar{z}^{2}-2 \bar{w} w \bar{z} & 3 w \bar{z}^{2} \\
-\bar{w}^{3} & \bar{w}^{2} \bar{z} & -\overline{w z}^{2} & \bar{z}^{3}
\end{array}\right] \\
& T_{4}=\left[\begin{array}{ccccc}
z^{4} & z^{3} w & z^{2} w^{2} & z w^{3} & w^{4} \\
-4 z^{3} \bar{w} & z^{3} \bar{z}-3 \bar{w} z^{2} w & 2 z^{2} w \bar{z}-2 \bar{w} z w^{2} & 3 z w^{2} \bar{z}-\bar{w} w^{3} & 4 w^{3} \bar{z} \\
6 z^{2} \bar{w}^{2} & -3 z^{2} \overline{w z}+3 z \bar{w}^{2} w & z^{2} \bar{z}^{2}-4 z \bar{w} w \bar{z}+\bar{w}^{2} w^{2} & 3 z w \bar{z}^{2}-3 \bar{w} w^{2} \bar{z} & 6 w^{2} \bar{z}^{2} \\
-4 z \bar{w}^{3} & 3 z \bar{w}^{2} \bar{z}-\bar{w}^{3} w & -2 z \overline{w z}^{2}+2 \bar{w}^{2} w \bar{z} & z \bar{z}^{3}-3 \bar{w} w \bar{z}^{2} & 4 w \bar{z}^{3} \\
\bar{w}^{4} & -\bar{w}^{3} \bar{z} & \bar{w}^{2} \bar{z}^{2} & -\overline{w z}^{3} & \bar{z}^{4}
\end{array}\right] \\
& O_{0}=\left[\frac{1}{|x|^{2}}\right] \\
& O_{1}=\frac{1}{|x|^{4}}\left[\begin{array}{cc}
\bar{z} & -w \\
\bar{w} & z
\end{array}\right] \\
& O_{2}=\frac{1}{|x|^{6}}\left[\begin{array}{ccc}
\bar{z}^{2} & -\bar{z} w & w^{2} \\
2 \overline{z w} & \bar{z} z-\bar{w} w & -2 w z \\
\bar{w}^{2} & \bar{w} z & z^{2}
\end{array}\right] \\
& O_{3}=\frac{1}{|x|^{8}}\left[\begin{array}{cccc}
\bar{z}^{3} & -\bar{z}^{2} w & \bar{z} w^{2} & -w^{3} \\
3 \bar{z}^{2} \bar{w} & z \bar{z}^{2}-2 \overline{w z} w & -2 \bar{z} w z+\bar{w} w^{2} & 3 w^{2} z \\
3 \overline{z w}^{2} & 2 \overline{z w} z-\bar{w}^{2} w & \bar{z} z^{2}-2 \bar{w} w z & -3 w z^{2} \\
\bar{w}^{3} & \bar{w}^{2} z & \bar{w} z^{2} & z^{3}
\end{array}\right] \\
& O_{4}=\frac{1}{|x|^{10}}\left[\begin{array}{ccccc}
\bar{z}^{4} & -\bar{z}^{3} w & \bar{z}^{2} w^{2} & -\bar{z} w^{3} & w^{4} \\
4 \bar{z}^{3} \bar{w} & z \bar{z}^{3}-3 \overline{w z}^{2} w & -2 z \bar{z}^{2} w+2 \overline{w z} w^{2} & 3 \bar{z} w^{2} z-\bar{w} w^{3} & -4 w^{3} z \\
6 \bar{z}^{2} \bar{w}^{2} & 3 z \bar{z}^{2} \bar{w}-3 \overline{z w}^{2} w & \bar{z}^{2} z^{2}-4 \overline{z w} w z+\bar{w}^{2} w^{2} & -3 \bar{z} w z^{2}+3 \bar{w} w^{2} z & 6 w^{2} z^{2} \\
4 \overline{z w}^{3} & 3 \overline{z w}^{2} z-\bar{w}^{3} w & 2 \overline{z w} z^{2}-2 \bar{w}^{2} w z & \bar{z} z^{3}-3 \bar{w} w z^{2} & -4 w z^{3} \\
\bar{w}^{4} & \bar{w}^{3} z & \bar{w}^{2} z^{2} & \bar{w} z^{3} & z^{4}
\end{array}\right] \\
& |x|^{2}=z \bar{z}+w \bar{w}
\end{aligned}
$$

